\title{
Special Lagrangian fibrations, wall-crossing, and mirror symmetry
}

\author{
Denis Auroux
}

\begin{abstract}
In this survey paper, we briefly review various aspects of the SYZ approach to mirror symmetry for non-Calabi-Yau varieties, focusing in particular on Lagrangian fibrations and wall-crossing phenomena in Floer homology. Various examples are presented, some of them new.
\end{abstract}

\section{Contents}

1. Introduction 2

2. Lagrangian tori and mirror symmetry 3

2.1. Lagrangian tori and the SYZ conjecture 3

2.2. Beyond the Calabi-Yau case: Landau-Ginzburg models 6

2.3. Example: Fano toric varieties 10

3. Examples of wall-crossing and instanton corrections 12

3.1. First examples 12

3.2. Beyond the Fano case: Hirzebruch surfaces 16

3.3. Higher dimensions 24

4. Floer-theoretic considerations 30

4.1. Deformations and local systems 30

4.2. Failure of invariance and divergence issues 34

5. Relative mirror symmetry 38

$\begin{array}{ll}\text { 5.1. Mirror symmetry for pairs } & 38\end{array}$

5.2. Homological mirror symmetry 41

5.3. Complete intersections 44

References $\quad 45$

This work was partially supported by NSF grants DMS-0600148 and DMS-0652630.

(C)2009 International Press 


\section{Introduction}

While mirror symmetry first arose as a set of predictions relating Hodge structures and quantum cohomology for Calabi-Yau 3-folds (see e.g. $[\mathbf{8}, \mathbf{1 3}]$ ), it has since been extended in spectacular ways. To mention just a few key advances, Kontsevich's homological mirror conjecture [26] has recast mirror symmetry in the language of derived categories of coherent sheaves and Fukaya categories; the Strominger-Yau-Zaslow (SYZ) conjecture [39] has provided the basis for a geometric understanding of mirror symmetry; and mirror symmetry has been extended beyond the Calabi-Yau setting, by considering Landau-Ginzburg models (see e.g. $[\mathbf{2 3}, \mathbf{2 7}]$ ).

In this paper, we briefly discuss various aspects of mirror symmetry from the perspective of Lagrangian torus fibrations, i.e. following the StromingerYau-Zaslow philosophy [39]. We mostly focus on the case of Kähler manifolds with effective anticanonical divisors, along the same general lines as [4]. The two main phenomena that we would like to focus on here are, on one hand, wall-crossing in Floer homology and its role in determining "instanton corrections" to the complex geometry of the mirror; and on the other hand, the possibility of "transferring" mirror symmetry from a given Kähler manifold to a Calabi-Yau submanifold.

The paper is essentially expository in nature, expanding on the themes already present in [4]. The discussion falls far short of the level of sophistication present in the works of Kontsevich-Soibelman [28, 29], Gross-Siebert $[\mathbf{1 8}, \mathbf{1 9}]$, or Fukaya-Oh-Ohta-Ono $[\mathbf{1 4}, \mathbf{1 5}]$; rather, our goal is to show how various important ideas in the modern understanding of mirror symmetry naturally arise from the perspective of a symplectic geometer, and to illustrate them by simple examples. Accordingly, most of the results mentioned here are not new, though to our knowledge some of them have not appeared anywhere in the literature.

Another word of warning is in order: we have swept under the rug many of the issues related to the rigorous construction of Lagrangian Floer theory, and generally speaking we take an optimistic view of issues such as the existence of fundamental chains for moduli spaces of discs and the convergence of various Floer-theoretic quantities. These happen not to be issues in the examples we consider, but can be serious obstacles in the general case.

The rest of this paper is organized as follows: in Section 2 we review the SYZ approach to the construction of mirror pairs, and the manner in which the mirror superpotential arises naturally as a Floer-theoretic obstruction in the non Calabi-Yau case. Section 3 presents various elementary examples, focusing on wall-crossing phenomena and instanton corrections. Section 4 discusses some issues related to convergent power series Floer homology. Finally, Section 5 focuses on mirror symmetry in the relative setting, namely for a Calabi-Yau hypersurface representing the anticanonical class inside a Kähler manifold, or more generally for a complete intersection. 
Acknowledgements. The ideas presented here were influenced in a decisive manner by numerous discussions with Mohammed Abouzaid, Paul Seidel, and Ludmil Katzarkov. Some of the topics presented here also owe a lot to conversations with Dima Orlov, Mark Gross, and Kenji Fukaya. Finally, I am grateful to Mohammed Abouzaid for valuable comments on the exposition. This work was partially supported by NSF grants DMS-0600148 and DMS-0652630.

\section{Lagrangian tori and mirror symmetry}

2.1. Lagrangian tori and the SYZ conjecture. The SYZ conjecture essentially asserts that mirror pairs of Calabi-Yau manifolds should carry dual special Lagrangian torus fibrations [39]. This statement should be understood with suitable qualifiers (near the large complex structure limit, with instanton corrections, etc.), but it nonetheless gives the basic template for the geometric construction of mirror pairs.

From this perspective, to construct the mirror of a given Calabi-Yau manifold $X$, one should first try to construct a special Lagrangian torus fibration $f: X \rightarrow B$. This is a difficult problem, but assuming it has been solved, the first guess for the mirror manifold $X^{\vee}$ is then the total space of the dual fibration $f^{\vee}$. Given a torus $T$, the dual torus $T^{\vee}=\operatorname{Hom}\left(\pi_{1}(T), S^{1}\right)$ can be viewed as a moduli space of rank 1 unitary local systems (i.e., flat unitary connections up to gauge equivalence) over $T$; hence, points of the dual fibration parametrize pairs consisting of a special Lagrangian fiber in $X$ and a unitary local system over it.

More precisely, let $(X, J, \omega)$ be a Kähler manifold of complex dimension $n$, equipped with a nonvanishing holomorphic volume form $\Omega \in \Omega^{n, 0}(X)$. This is sometimes called an "almost Calabi-Yau" manifold (to distinguish it from a genuine Calabi-Yau, where one would also require the norm of $\Omega$ with respect to the Kähler metric to be constant). It is an elementary fact that the restriction of $\Omega$ to a Lagrangian submanifold $L \subset X$ is a nowhere vanishing complex-valued $n$-form.

Definition 2.1. A Lagrangian submanifold $L \subset X$ is special Lagrangian if the argument of $\Omega_{\mid L}$ is constant.

The value of the constant depends only on the homology class of $L$, and we will usually normalize $\Omega$ so that it is a multiple of $\pi / 2$. For simplicity, in the rest of this paragraph we will assume that $\Omega_{\mid L}$ is a positive real multiple of the real volume form $\operatorname{vol}_{g}$ induced by the Kähler metric $g=\omega(\cdot, J \cdot)$.

The following classical result is due to McLean [32] (in the Calabi-Yau setting; see $\S 9$ of [24] or Proposition 2.5 of [4] for the almost Calabi-Yau case):

Proposition 2.2 (McLean). Infinitesimal special Lagrangian deformations of $L$ are in one to one correspondence with cohomology classes in $H^{1}(L, \mathbb{R})$. Moreover, the deformations are unobstructed. 
Specifically, a section of the normal bundle $v \in C^{\infty}(N L)$ determines a 1 -form $\alpha=-\iota_{v} \omega \in \Omega^{1}(L, \mathbb{R})$ and an $(n-1)$-form $\beta=\iota_{v} \operatorname{Im} \Omega \in \Omega^{n-1}(L, \mathbb{R})$. These satisfy $\beta=\psi *_{g} \alpha$, where $\psi \in C^{\infty}\left(L, \mathbb{R}_{+}\right)$is the ratio between the volume elements determined by $\Omega$ and $g$, i.e. the norm of $\Omega$ with respect to the Kähler metric; moreover, the deformation is special Lagrangian if and only if $\alpha$ and $\beta$ are both closed. Thus special Lagrangian deformations correspond to " $\psi$-harmonic" 1-forms

$$
-\iota_{v} \omega \in \mathcal{H}_{\psi}^{1}(L)=\left\{\alpha \in \Omega^{1}(L, \mathbb{R}) \mid d \alpha=0, d^{*}(\psi \alpha)=0\right\} .
$$

In particular, special Lagrangian tori occur in $n$-dimensional families, giving a local fibration structure provided that nontrivial $\psi$-harmonic 1 -forms have no zeroes.

The base $B$ of a special Lagrangian torus fibration carries two natural affine structures, which we call "symplectic" and "complex". The first one, which encodes the symplectic geometry of $X$, locally identifies $B$ with a domain in $H^{1}(L, \mathbb{R})\left(L \approx T^{n}\right)$. At the level of tangent spaces, the cohomology class of $-\iota_{v} \omega$ provides an identification of $T B$ with $H^{1}(L, \mathbb{R})$; integrating, the local affine coordinates on $B$ are the symplectic areas swept by loops forming a basis of $H_{1}(L)$. The other affine structure encodes the complex geometry of $X$, and locally identifies $B$ with a domain in $H^{n-1}(L, \mathbb{R})$. Namely, one uses the cohomology class of $\iota_{v} \operatorname{Im} \Omega$ to identify $T B$ with $H^{n-1}(L, \mathbb{R})$, and the affine coordinates are obtained by integrating $\operatorname{Im} \Omega$ over the $n$-chains swept by cycles forming a basis of $H_{n-1}(L)$.

The dual special Lagrangian fibration can be constructed as a moduli space $M$ of pairs $(L, \nabla)$, where $L \subset X$ is a special Lagrangian fiber and $\nabla$ is a rank 1 unitary local system over $L$. The local geometry of $M$ is wellunderstood (cf. e.g. $[\mathbf{2 0}, \mathbf{3 0}, \mathbf{1 7}]$ ); in particular we have the following result (cf. e.g. $\S 2$ of $[4])$ :

Proposition 2.3. Let $M$ be the moduli space of pairs $(L, \nabla)$, where $L$ is a special Lagrangian torus in $X$ and $\nabla$ is a flat $U(1)$ connection on the trivial complex line bundle over $L$ up to gauge. Then $M$ carries a natural integrable complex structure $J^{\vee}$ arising from the identification

$$
T_{(L, \nabla)} M=\left\{(v, \alpha) \in C^{\infty}(N L) \oplus \Omega^{1}(L, \mathbb{R}) \mid-\iota_{v} \omega+i \alpha \in \mathcal{H}_{\psi}^{1}(L) \otimes \mathbb{C}\right\},
$$

a holomorphic $n$-form

$$
\Omega^{\vee}\left(\left(v_{1}, \alpha_{1}\right), \ldots,\left(v_{n}, \alpha_{n}\right)\right)=\int_{L}\left(-\iota_{v_{1}} \omega+i \alpha_{1}\right) \wedge \cdots \wedge\left(-\iota_{v_{n}} \omega+i \alpha_{n}\right),
$$

and a compatible Kähler form

$$
\omega^{\vee}\left(\left(v_{1}, \alpha_{1}\right),\left(v_{2}, \alpha_{2}\right)\right)=\int_{L} \alpha_{2} \wedge \iota_{v_{1}} \operatorname{Im} \Omega-\alpha_{1} \wedge \iota_{v_{2}} \operatorname{Im} \Omega
$$

(this formula for $\omega^{\vee}$ assumes that $\int_{L} \operatorname{Re} \Omega$ has been suitably normalized). 
In particular, $M$ can be viewed as a complexification of the moduli space of special Lagrangian submanifolds; forgetting the connection gives a projection map $f^{\vee}$ from $M$ to the real moduli space $B$. The fibers of this projection are easily checked to be special Lagrangian tori in the almost Calabi-Yau manifold $\left(M, J^{\vee}, \omega^{\vee}, \Omega^{\vee}\right)$.

This special Lagrangian fibration on $M$ is fiberwise dual to the one previously considered on $X$; they have the same base $B$, and passing from one fibration to the other simply amounts to exchanging the roles of the two affine structures on $B$.

In real life, unless we restrict ourselves to complex tori, we have to consider special Lagrangian torus fibrations with singularities. The base of the fibration is then a singular affine manifold, and the picture discussed above only holds away from the singularities. A natural idea would be to obtain the mirror by first constructing the dual fibration away from the singularities, and then trying to extend it over the singular locus. Unfortunately, this cannot be done directly; instead we need to modify the complex geometry of $M$ by introducing instanton corrections.

To give some insight into the geometric meaning of these corrections, consider the SYZ conjecture from the perspective of homological mirror symmetry.

Recall that Kontsevich's homological mirror symmetry conjecture [26] predicts that the derived category of coherent sheaves $D^{b} \operatorname{Coh}\left(X^{\vee}\right)$ of the mirror $X^{\vee}$ is equivalent to the derived Fukaya category of $X$. For any point $p \in X^{\vee}$, the skyscraper sheaf $\mathcal{O}_{p}$ is an object of the derived category. Since $\operatorname{Ext}^{*}\left(\mathcal{O}_{p}, \mathcal{O}_{p}\right) \simeq H^{*}\left(T^{n} ; \mathbb{C}\right)$ (as a graded vector space), we expect that $\mathcal{O}_{p}$ corresponds to some object $\mathcal{L}_{p}$ of the derived Fukaya category of $X$ such that $\operatorname{End}\left(\mathcal{L}_{p}\right) \simeq H^{*}\left(T^{n}\right)$. It is natural to conjecture that, generically, the object $\mathcal{L}_{p}$ is a Lagrangian torus in $X$ with trivial Maslov class, equipped with a rank 1 unitary local system, and such that $H F^{*}\left(\mathcal{L}_{p}, \mathcal{L}_{p}\right) \simeq H^{*}\left(T^{n}\right)$ (as a graded vector space). This suggests constructing the mirror $X^{\vee}$ as a moduli space of such objects of the Fukaya category of $X$. (However it could still be the case that some points of $X^{\vee}$ cannot be realized by honest Lagrangian tori in $X$.)

In the Calabi-Yau setting, it is expected that "generically" (i.e., subject to a certain stability condition) the Hamiltonian isotopy class of the Lagrangian torus $\mathcal{L}_{p}$ should contain a unique special Lagrangian representative $[\mathbf{4 0 , 4 1}$. Hence it is natural to restrict one's attention to special Lagrangians, whose geometry is richer than that of Lagrangians: for instance, the moduli space considered in Proposition 2.3 carries not only a complex structure, but also a symplectic structure. However, if we only care about the complex geometry of the mirror $X^{\vee}$ and not its symplectic geometry, then it should not be necessary to consider special Lagrangians.

On the other hand, due to wall-crossing phenomena, the "convergent power series" version of Lagrangian Floer homology which is directly relevant to the situation here is not quite invariant under Hamiltonian isotopies 
(see e.g. [10], and Section 4 below). Hence, we need to consider a corrected equivalence relation on the moduli space of Lagrangian tori in $X$ equipped with unitary local systems. Loosely speaking, we'd like to say that two Lagrangian tori (equipped unitary local systems) are equivalent if they behave interchangeably with respect to convergent power series Floer homology; however, giving a precise meaning to this statement is rather tricky.

2.2. Beyond the Calabi-Yau case: Landau-Ginzburg models. Assume now that $(X, J, \omega)$ is a Kähler manifold of complex dimension $n$, and that $D \subset X$ is an effective divisor representing the anticanonical class, with at most normal crossing singularities. Then the inverse of the defining section of $D$ is a section of the canonical line bundle $K_{X}$ over $X \backslash D$, i.e. a holomorphic volume form $\Omega \in \Omega^{n, 0}(X \backslash D)$ with simple poles along $D$.

We can try to construct a mirror to the almost Calabi-Yau manifold $X \backslash D$ just as above, by considering a suitable moduli space of (special) Lagrangian tori in $X \backslash D$ equipped with unitary local systems. The assumption on the behavior of $\Omega$ near $D$ is necessary for the existence of a special Lagrangian torus fibration with the desired properties: for instance, a neighborhood of the origin in $\mathbb{C}$ equipped with $\Omega=z^{k} d z$ does not contain any compact special Lagrangians unless $k=-1$.

Compared to $X \backslash D$, the manifold $X$ contains essentially the same Lagrangians. However, (special) Lagrangian tori in $X \backslash D$ typically bound families of holomorphic discs in $X$, which causes their Floer homology to be obstructed in the sense of Fukaya-Oh-Ohta-Ono [14]. Namely, Floer theory associates to $\mathcal{L}=(L, \nabla)$ (where $L$ is a Lagrangian torus in $X \backslash D$ and $\nabla$ is a flat $U(1)$ connection on the trivial line bundle over $L$ ) an element $\mathfrak{m}_{0}(\mathcal{L}) \in C F^{*}(\mathcal{L}, \mathcal{L})$, given by a weighted count of holomorphic discs in $(X, L)$.

More precisely, recall that in Fukaya-Oh-Ohta-Ono's approach the Floer complex $C F^{*}(\mathcal{L}, \mathcal{L})$ is generated by chains on $L$ (with suitable coefficients), and its element $\mathfrak{m}_{0}(\mathcal{L})$ is defined as follows (see [14] for details). Given a class $\beta \in \pi_{2}(X, L)$, the moduli space $\mathcal{M}_{k}(L, \beta)$ of holomorphic discs in $(X, L)$ with $k$ boundary marked points representing the class $\beta$ has expected dimension $n-3+k+\mu(\beta)$, where $\mu(\beta)$ is the Maslov index; when $L \subset X \backslash D$ is special Lagrangian, $\mu(\beta)$ is simply twice the algebraic intersection number $\beta \cdot[D]$ (see e.g. Lemma 3.1 of [4]). This moduli space can be compactified by adding bubbled configurations. Assuming regularity, this yields a manifold with boundary, which carries a fundamental chain $\left[\overline{\mathcal{M}}_{k}(L, \beta)\right]$; otherwise, various techniques can be used to define a virtual fundamental chain $\left[\overline{\mathcal{M}}_{k}(L, \beta)\right]^{\text {vir }}$, usually dependent on auxiliary perturbation data. The (virtual) fundamental chain of $\overline{\mathcal{M}}_{1}(L, \beta)$ can be pushed forward by the evaluation map at the marked point, ev $: \overline{\mathcal{M}}_{1}(L, \beta) \rightarrow L$, to obtain a chain in $L$ : then one sets

$$
\mathfrak{m}_{0}(\mathcal{L})=\sum_{\beta \in \pi_{2}(X, L)} z_{\beta}(\mathcal{L}) e v_{*}\left[\overline{\mathcal{M}}_{1}(L, \beta)\right]^{\text {vir }},
$$


where the coefficient $z_{\beta}(\mathcal{L})$ reflects weighting by symplectic area:

$$
z_{\beta}(\mathcal{L})=\exp \left(-\int_{\beta} \omega\right) \operatorname{hol}_{\nabla}(\partial \beta) \in \mathbb{C}^{*}
$$

or $z_{\beta}(\mathcal{L})=T^{\int_{\beta} \omega} \operatorname{hol}_{\nabla}(\partial \beta) \in \Lambda_{0}$ if using Novikov coefficients to avoid convergence issues (see below).

Note that $z_{\beta}$ as defined by (2.2) is locally a holomorphic function with respect to the complex structure $J^{\vee}$ introduced in Proposition 2.3. Indeed, recall that the tangent space to the moduli space $M$ is identified with the space of complex-valued $\psi$-harmonic 1 -forms on $L$; the differential of $\log z_{\beta}$ is just the linear form on $\mathcal{H}_{\psi}^{1}(L) \otimes \mathbb{C}$ given by integration on the homology class $\partial \beta \in H_{1}(L)$.

In this paper we will mostly consider weakly unobstructed Lagrangians, i.e. those for which $\mathfrak{m}_{0}(\mathcal{L})$ is a multiple of the unit (the fundamental cycle of $L)$. In that case, the Floer differential on $C F^{*}(\mathcal{L}, \mathcal{L})$ does square to zero, but given two Lagrangians $\mathcal{L}, \mathcal{L}^{\prime}$ we find that $C F^{*}\left(\mathcal{L}, \mathcal{L}^{\prime}\right)$ may not be well-defined as a chain complex. To understand the obstruction, recall that the count of holomorphic triangles equips $C F^{*}\left(\mathcal{L}, \mathcal{L}^{\prime}\right)$ with the structure of a left module over $C F^{*}(\mathcal{L}, \mathcal{L})$ and a right module over $C F^{*}\left(\mathcal{L}^{\prime}, \mathcal{L}^{\prime}\right)$. Writing $\mathfrak{m}_{2}$ for both module maps, an analysis of the boundary of 1-dimensional moduli spaces shows that the differential on $C F^{*}\left(\mathcal{L}, \mathcal{L}^{\prime}\right)$ squares to

$$
\mathfrak{m}_{2}\left(\mathfrak{m}_{0}\left(\mathcal{L}^{\prime}\right), \cdot\right)-\mathfrak{m}_{2}\left(\cdot, \mathfrak{m}_{0}(\mathcal{L})\right) .
$$

The assumption that $\mathfrak{m}_{0}$ is a multiple of the identity implies that Floer homology is only defined for pairs of Lagrangians which have the same $\mathfrak{m}_{0}$. Moreover, even though the Floer homology group $H F^{*}(\mathcal{L}, \mathcal{L})$ can still be defined, it is generically zero due to contributions of holomorphic discs in $(X, L)$ to the Floer differential; in that case $\mathcal{L}$ is a trivial object of the Fukaya category.

On the mirror side, these features of the theory can be replicated by the introduction of a superpotential, i.e. a holomorphic function $W: X^{\vee} \rightarrow \mathbb{C}$ on the mirror of $X \backslash D$. W can be thought of as an obstruction term for the B-model on $X^{\vee}$, playing the same role as $\mathfrak{m}_{0}$ for the A-model on $X$. More precisely, homological mirror symmetry predicts that the derived Fukaya category of $X$ is equivalent to the derived category of singularities of the mirror Landau-Ginzburg model $\left(X^{\vee}, W\right)[\mathbf{2 5}, \mathbf{3 3}]$. This category is actually a collection of categories indexed by complex numbers, just as the derived Fukaya category of $X$ is a collection of categories indexed by the values of $\mathfrak{m}_{0}$.

Given $\lambda \in \mathbb{C}$, one defines $D_{\text {sing }}^{b}(W, \lambda)=D^{b} \operatorname{Coh}\left(W^{-1}(\lambda)\right) / \operatorname{Perf}\left(W^{-1}(\lambda)\right)$, the quotient of the derived category of coherent sheaves on the fiber $W^{-1}(\lambda)$ by the subcategory of perfect complexes. Since for smooth fibers the derived category of coherent sheaves is generated by vector bundles, this quotient is trivial unless $\lambda$ is a critical value of $W$; in particular, a point of $X^{\vee}$ defines a nontrivial object of the derived category of singularities only if it is a critical point of $W$. Alternatively, this category can also be defined in 
terms of matrix factorizations. Assuming $X^{\vee}$ to be affine for simplicity, a matrix factorization is a $\mathbb{Z} / 2$-graded projective $\mathbb{C}\left[X^{\vee}\right]$-module together with an odd endomorphism $\delta$ such that $\delta^{2}=(W-\lambda)$ id. For a fixed value of $\lambda$, matrix factorizations yield a $\mathbb{Z} / 2$-graded dg-category, whose cohomological category is equivalent to $D_{\text {sing }}^{b}(W, \lambda)$ by a result of Orlov [33]. However, if we consider two matrix factorizations $\left(P_{1}, \delta_{1}\right)$ and $\left(P_{2}, \delta_{2}\right)$ associated to two values $\lambda_{1}, \lambda_{2} \in \mathbb{C}$, then the differential on $\operatorname{hom}\left(\left(P_{1}, \delta_{1}\right),\left(P_{2}, \delta_{2}\right)\right)$ squares to $\left(\lambda_{1}-\lambda_{2}\right)$ id, similarly to the Floer differential on the Floer complex of two Lagrangians with different values of $\mathfrak{m}_{0}$.

This motivates the following conjecture:

ConjeCture 2.4. The mirror of $X$ is the Landau-Ginzburg model $\left(X^{\vee}, W\right)$, where

(1) $X^{\vee}$ is a mirror of the almost Calabi-Yau manifold $X \backslash D$, i.e. a (corrected and completed) moduli space of special Lagrangian tori in $X \backslash D$ equipped with rank 1 unitary local systems;

(2) $W: X^{\vee} \rightarrow \mathbb{C}$ is a holomorphic function defined as follows: if $p \in$ $X^{\vee}$ corresponds to a special Lagrangian $\mathcal{L}_{p}=(L, \nabla)$, then

$$
W(p)=\sum_{\beta \in \pi_{2}(X, L), \mu(\beta)=2} n_{\beta}\left(\mathcal{L}_{p}\right) z_{\beta}\left(\mathcal{L}_{p}\right),
$$

where $n_{\beta}\left(\mathcal{L}_{p}\right)$ is the degree of the evaluation chain ev $v_{*}\left[\overline{\mathcal{M}}_{1}(L, \beta)\right]^{\text {vir }}$, i.e., the (virtual) number of holomorphic discs in the class $\beta$ passing through a generic point of $L$, and the weight $z_{\beta}\left(\mathcal{L}_{p}\right)$ is given by (2.2).

There are several issues with the formula (2.3). To start with, except in specific cases (e.g. Fano toric varieties), there is no guarantee that the sum in (2.3) converges. The rigorous way to deal with this issue is to work over the Novikov ring

$$
\Lambda_{0}=\left\{\sum_{i} a_{i} T^{\lambda_{i}} \mid a_{i} \in \mathbb{C}, \lambda_{i} \in \mathbb{R}_{\geq 0}, \lambda_{i} \rightarrow+\infty\right\}
$$

rather than over complex numbers. Holomorphic discs in a class $\beta$ are then counted with weight $T^{\int_{\beta} \omega} \operatorname{hol}_{\nabla}(\partial \beta)$ instead of $\exp \left(-\int_{\beta} \omega\right) \operatorname{hol}_{\nabla}(\partial \beta)$. Assuming convergence, setting $T=e^{-1}$ recovers the complex coefficient version.

Morally, working over Novikov coefficients simultaneously encodes the family of mirrors for $X$ equipped with the family of Kähler forms $\kappa \omega, \kappa \in$ $\mathbb{R}_{+}$. Namely, the mirror manifold should be constructed as a variety defined over the Novikov field $\Lambda$ (the field of fractions of $\Lambda_{0}$ ), and the superpotential as a regular function with values in $\Lambda$. If convergence holds, then setting $T=\exp (-\kappa)$ recovers the complex mirror to $(X, \kappa \omega)$; if convergence fails for all values of $T$, the mirror might actually exist only in a formal sense near the large volume limit $\kappa \rightarrow \infty$. 
Another issue with Conjecture 2.4 is the definition of the numbers $n_{\beta}\left(\mathcal{L}_{p}\right)$. Roughly speaking, the value of the superpotential is meant to be "the coefficient of the fundamental chain $[L]$ in $\mathfrak{m}_{0}$ ". However, in real life not all Lagrangians are weakly unobstructed: due to bubbling of Maslov index 0 discs, for a given class $\beta$ with $\mu(\beta)=2$ the chain $e v_{*}\left[\overline{\mathcal{M}}_{1}(L, \beta)\right]^{\text {vir }}$ is in general not a cycle. Thus we can still define $n_{\beta}$ to be its "degree" (or multiplicity) at some point $q \in L$, but the answer depends on the choice of $q$. Alternatively, we can complete the chain to a cycle, e.g. by choosing a "weak bounding cochain" in the sense of Fukaya-Oh-Ohta-Ono [14], or more geometrically, by considering not only holomorphic discs but also holomorphic "clusters" in the sense of Cornea-Lalonde [12]; however, $n_{\beta}$ will then depend on some auxiliary data (in the cluster approach, a Morse function on $L$ ).

Even if we equip each $L$ with the appropriate auxiliary data (e.g. a base point or a Morse function), the numbers $n_{\beta}$ will typically vary in a discontinuous manner due to wall-crossing phenomena. However, recall that $X^{\vee}$ differs from the naive moduli space of Proposition 2.3 by instanton corrections. Namely, $X^{\vee}$ is more accurately described as a (completed) moduli space of Lagrangian tori $L \subset X \backslash D$ equipped with not only a $U(1)$ local system but also the auxiliary data needed to make sense of the Floer theory of $L$ in general and of the numbers $n_{\beta}$ in particular. The equivalence relation on this set of Lagrangians equipped with extra data is Floer-theoretic in nature. General considerations about wall-crossing and continuation maps in Floer theory imply that, even though the individual numbers $n_{\beta}$ depend on the choice of a representative in the equivalence class, by construction the superpotential $W$ given by (2.3) is a single-valued smooth function on the corrected moduli space. The reader is referred to $\S 19.1$ in [14] and $\S 3$ in [4] for details.

In this paper we will assume that things don't go completely wrong, namely that our Lagrangians are weakly unobstructed except when they lie near a certain collection of walls in the moduli space. In this case, the process which yields the corrected moduli space from the naive one can be thought of decomposing $M$ into chambers over which the $n_{\beta}$ are locally constant, and gluing these chambers by analytic changes of coordinates dictated by the enumerative geometry of Maslov index 0 discs on the wall. Thus, the analyticity of $W$ on the corrected mirror follows from that of $z_{\beta}$ on the uncorrected moduli space.

One last thing to mention is that the incompleteness of the Kähler metric on $X \backslash D$ causes the moduli space of Lagrangians to be similarly incomplete. This is readily apparent if we observe that, since $\left|z_{\beta}\right|=\exp \left(-\int_{\beta} \omega\right)$, each variable $z_{\beta}$ appearing in the sum (2.3) takes values in the unit disc. We will want to define the mirror of $X$ to be a larger space, obtained by analytic continuation of the instanton-corrected moduli space of Lagrangian tori (i.e., roughly speaking, allowing $\left|z_{\beta}\right|$ to be arbitrarily large). One way to think of the points of $X^{\vee}$ added in the completion process is as Lagrangian tori in $X \backslash D$ equipped with non-unitary local systems; however this can lead to serious convergence issues, even when working over the Novikov ring. 
Another way to think about the completion process, under the assumption that $D$ is nef, is in terms of inflating $X$ along $D$, i.e replacing the Kähler form $\omega$ by $\omega_{t}=\omega+t \eta$ where the $(1,1)$-form $\eta$ is Poincaré dual to $D$ and supported in a neighborhood of $D$; this "enlarges" the moduli space of Lagrangians near $D$, and simultaneously increases the area of all Maslov index 2 discs by $t$, i.e. rescales the superpotential by a factor of $e^{-t}$. Taking the limit as $t \rightarrow \infty$ (and rescaling the superpotential appropriately) yields the completed mirror.

2.3. Example: Fano toric varieties. Let $(X, \omega, J)$ be a smooth toric variety of complex dimension $n$. In this section we additionally assume that $X$ is Fano, i.e. its anticanonical divisor is ample. As a Kähler manifold, $X$ is determined by its moment polytope $\Delta \subset \mathbb{R}^{n}$, a convex polytope in which every facet admits an integer normal vector, $n$ facets meet at every vertex, and their primitive integer normal vectors form a basis of $\mathbb{Z}^{n}$. The moment map $\phi: X \rightarrow \mathbb{R}^{n}$ identifies the orbit space of the $T^{n}$-action on $X$ with $\Delta$. From the point of view of complex geometry, the preimage of the interior of $\Delta$ is an open dense subset $U$ of $X$, biholomorphic to $\left(\mathbb{C}^{*}\right)^{n}$, on which $T^{n}=\left(S^{1}\right)^{n}$ acts in the standard manner. Moreover $X$ admits an open cover by affine subsets biholomorphic to $\mathbb{C}^{n}$, which are the preimages of the open stars of the vertices of $\Delta$ (i.e., the union of all the strata whose closure contains the given vertex).

For each facet $F$ of $\Delta$, the preimage $\phi^{-1}(F)=D_{F}$ is a hypersurface in $X$; the union of these hypersurfaces defines the toric anticanonical divisor $D=\sum_{F} D_{F}$. The standard holomorphic volume form on $\left(\mathbb{C}^{*}\right)^{n} \simeq U=$ $X \backslash D$, defined in coordinates by $\Omega=d \log x_{1} \wedge \cdots \wedge d \log x_{n}$, determines a section of $K_{X}$ with poles along $D$.

It is straightforward to check that the orbits of the $T^{n}$-action are special Lagrangian with respect to $\omega$ and $\Omega$. Thus the moment map determines a special Lagrangian fibration on $X \backslash D$, with base $B=\operatorname{int}(\Delta)$; by definition, the symplectic affine structure induced on $B$ by the identification $T B \approx$ $H^{1}(L, \mathbb{R})$ is precisely the standard one coming from the inclusion of $B$ in $\mathbb{R}^{n}$ (up to a scaling factor of $2 \pi$ ).

Consider a $T^{n}$-orbit $L$ in the open stratum $X \backslash D \approx\left(\mathbb{C}^{*}\right)^{n}$, and a flat $U(1)$-connection $\nabla$ on the trivial bundle over $L$. Let

$$
z_{j}(L, \nabla)=\exp \left(-2 \pi \phi_{j}(L)\right) \operatorname{hol}_{\nabla}\left(\gamma_{j}\right)
$$

where $\phi_{j}$ is the $j$-th component of the moment map, i.e. the Hamiltonian for the action of the $j$-th factor of $T^{n}$, and $\gamma_{j}=\left[S^{1}\left(r_{j}\right)\right] \in H_{1}(L)$ is the homology class corresponding to the $j$-th factor in $L=S^{1}\left(r_{1}\right) \times \cdots \times S^{1}\left(r_{n}\right) \subset\left(\mathbb{C}^{*}\right)^{n}$. Then $z_{1}, \ldots, z_{n}$ are holomorphic coordinates on the moduli space $M$ of pairs $(L, \nabla)$ equipped with the complex structure $J^{\vee}$ of Proposition 2.3.

For each facet $F$ of $\Delta$, denote by $\nu(F) \in \mathbb{Z}^{n}$ the primitive integer normal vector to $F$ pointing into $\Delta$, and let $\alpha(F) \in \mathbb{R}$ be the constant such that the 
equation of $F$ is $\langle\nu(F), \phi\rangle+\alpha(F)=0$. Moreover, given $a=\left(a_{1}, \ldots, a_{n}\right) \in \mathbb{Z}^{n}$ we denote by $z^{a}$ the Laurent monomial $z_{1}^{a_{1}} \ldots z_{n}^{a_{n}}$.

Proposition 2.5. The $S Y Z$ mirror to the smooth Fano toric variety $X$ is $\left(\mathbb{C}^{*}\right)^{n}$ equipped with a superpotential given by the Laurent polynomial

$$
W=\sum_{F \text { facet }} e^{-2 \pi \alpha(F)} z^{\nu(F)} .
$$

More precisely, the moduli space $M$ of pairs $(L, \nabla)$ is biholomorphic to the bounded open subset of $\left(\mathbb{C}^{*}\right)^{n}$ consisting of all points $\left(z_{1}, \ldots, z_{n}\right)$ such that each term in the sum (2.5) has norm less than 1; however, the completed mirror is all of $\left(\mathbb{C}^{*}\right)^{n}$.

Proposition 2.5 is a well-known result, which appears in many places; for completeness we give a very brief sketch of a geometric proof (see also $[21, \mathbf{1 1}, 4,15]$ for more details).

Sketch OF PROOF. Consider a pair $(L, \nabla)$ as above, and recall that $L$ can be identified with a product torus $S^{1}\left(r_{1}\right) \times \cdots \times S^{1}\left(r_{n}\right)$ inside $\left(\mathbb{C}^{*}\right)^{n}$. It follows from the maximum principle that $L$ does not bound any nonconstant holomorphic discs in $\left(\mathbb{C}^{*}\right)^{n}$; since the Maslov index of a disc in $(X, L)$ is twice its intersection number with the toric divisor $D$, this eliminates the possibility of Maslov index 0 discs. Moreover, since $X$ is Fano, all holomorphic spheres in $X$ have positive Chern number. It follows that the moduli spaces of Maslov index 2 holomorphic discs in $(X, L)$ are all compact, and that we do not have to worry about possible contributions from bubble trees of total Maslov index 2; this is in sharp contrast with the non-Fano case, see $\S 3.2$.

A holomorphic disc of Maslov index 2 in $(X, L)$ intersects $D$ at a single point, and in particular it intersects only one of the components, say $D_{F}$ for some facet $F$ of $\Delta$. Cho and $\mathrm{Oh}[\mathbf{1 1}]$ observed that for each facet $F$ there is a unique such disc whose boundary passes through a given point $\mathbf{x}^{0}=\left(x_{1}^{0}, \ldots, x_{n}^{0}\right) \in L$; in terms of the components $\left(\nu_{1}, \ldots, \nu_{n}\right)$ of the normal vector $\nu(F)$, this disc can be parametrized by the map

$$
w \mapsto\left(w^{\nu_{1}} x_{1}^{0}, \ldots, w^{\nu_{n}} x_{n}^{0}\right)
$$

(for $w \in D^{2} \backslash\{0\}$; the point $w=0$ corresponds to the intersection with $D_{F}$ ).

This is easiest to check in the model case where $X=\mathbb{C}^{n}$, the moment polytope is the positive octant $\mathbb{R}_{\geq 0}^{n}$, and the normal vectors to the facets form the standard basis of $\mathbb{Z}^{n}$. Namely, the maximum principle implies that holomorphic discs of Maslov index 2 with boundary in a product torus in $\mathbb{C}^{n}$ are given by maps with only one non-constant component, and up to reparametrization that non-constant component can be assumed to be linear. The general case is proved by working in an affine chart centered at a vertex of $\Delta$ adjacent to the considered facet $F$, and using a suitable change of coordinates to reduce to the previous case. 
A careful calculation shows that the map (2.6) is regular, and that its contribution to the signed count of holomorphic discs is +1 . Moreover, it follows from the definition of the moment map that the symplectic area of this disc is $2 \pi(\langle\nu(F), \phi(L)\rangle+\alpha(F))$. (This is again easiest to check in the model case of $\mathbb{C}^{n}$; the general case follows by performing a suitable change of coodinates). Exponentiating and multiplying by the appropriate holonomy factor, one arrives at (2.5).

Finally, recall that the interior of $\Delta$ is defined by the inequalities $\langle\nu(F)$, $\phi(L)\rangle+\alpha(F)>0$ for every facet $F$; exponentiating, this corresponds exactly to the constraint that $\left|e^{-2 \pi \alpha(F)} z^{\nu(F)}\right|<1$ for every facet $F$. However, adding the Poincaré dual of $t D$ to $\omega$ enlarges the moment polytope by $t / 2 \pi$ in every direction, i.e. it increases $\alpha(F)$ by $t / 2 \pi$ for all facets. This makes $M$ a larger subset of $\left(\mathbb{C}^{*}\right)^{n}$; rescaling $W$ by $e^{t}$ and taking the limit as $t \rightarrow+\infty$, we obtain all of $\left(\mathbb{C}^{*}\right)^{n}$ as claimed.

\section{Examples of wall-crossing and instanton corrections}

3.1. First examples. In this section we give two simple examples illustrating the construction of the mirror and the process of instanton corrections. The first example is explained in detail in $\S 5$ of $[\mathbf{4}]$, while the second example is the starting point of [2]; the two examples are in fact very similar.

ExAmple 3.1.1. Consider $X=\mathbb{C}^{2}$, equipped with a toric Kähler form $\omega$ and the holomorphic volume form $\Omega=d x \wedge d y /(x y-\epsilon)$, which has poles along the conic $D=\{x y=\epsilon\}$. Then $X \backslash D$ carries a fibration by special Lagrangian tori

$$
T_{r, \lambda}=\left\{(x, y) \in \mathbb{C}^{2},|x y-\epsilon|=r, \mu_{S^{1}}(x, y)=\lambda\right\},
$$

where $\mu_{S^{1}}$ is the moment map for the $S^{1}$-action $e^{i \theta} \cdot(x, y)=\left(e^{i \theta} x, e^{-i \theta} y\right)$, for instance $\mu_{S^{1}}(x, y)=\frac{1}{2}\left(|x|^{2}-|y|^{2}\right)$ for $\omega=\frac{i}{2}(d x \wedge d \bar{x}+d y \wedge d \bar{y})$. These tori are most easily visualized in terms of the projection $f:(x, y) \mapsto x y$, whose fibers are affine conics, each of which carries a $S^{1}$-action. The torus $T_{r, \lambda}$ lies in the preimage by $f$ of a circle of radius $r$ centered at $\epsilon$, and consists of a single $S^{1}$-orbit inside each fiber. In particular, $T_{r, \lambda}$ is smooth unless $(r, \lambda)=(|\epsilon|, 0)$, where we have a nodal singularity at the origin. One can check that $T_{r, \lambda}$ is special Lagrangian either by direct calculation, or by observing that $T_{r, \lambda}$ is the lift of a special Lagrangian circle in the reduced space $X_{r e d, \lambda}=\mu_{S^{1}}^{-1}(\lambda) / S^{1}$ equipped with the reduced Kähler form $\omega_{\text {red, } \lambda}$ and the reduced holomorphic volume form $\Omega_{r e d, \lambda}=\iota_{(\partial / \partial \theta) \#} \Omega=i d \log (x y-\epsilon)$; see $\S 5$ of $[4]$.

As seen in $\S 2$, away from $(r, \lambda)=(|\epsilon|, 0)$ the moduli space $M$ of pairs consisting of a torus $L=T_{r, \lambda}$ and a $U(1)$ local system $\nabla$ carries a natural complex structure, for which the functions $z_{\beta}=\exp \left(-\int_{\beta} \omega\right) \operatorname{hol}_{\nabla}(\partial \beta), \beta \in$ $\pi_{2}(X, L)$ are holomorphic. 


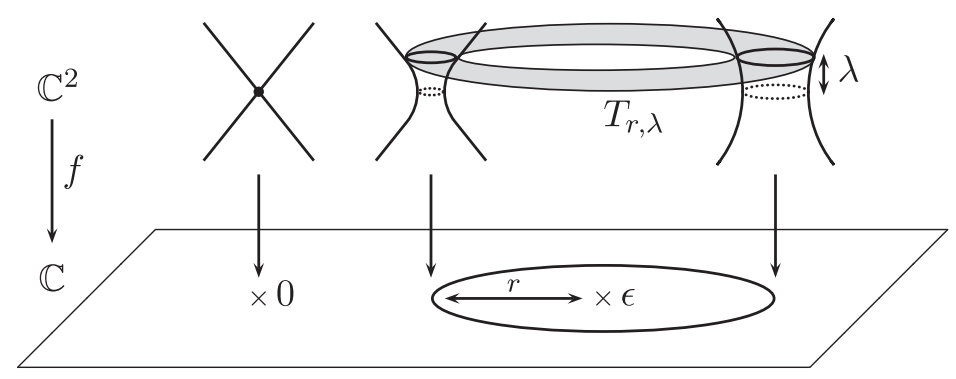

Figure 1. The special Lagrangian torus $T_{r, \lambda}$ in $\mathbb{C}^{2} \backslash D$

Wall-crossing occurs at $r=|\epsilon|$, namely the tori $T_{|\epsilon|, \lambda}$ for $\lambda>0$ intersect the $x$-axis in a circle, and thus bound a holomorphic disc contained in the fiber $f^{-1}(0)$, which has Maslov index 0 . Denote by $\alpha$ the relative homotopy class of this disc, and by $w=z_{\alpha}$ the corresponding holomorphic weight, which satisfies $|w|=e^{-\lambda}$. Similarly the tori $T_{|\epsilon|, \lambda}$ for $\lambda<0$ bound a holomorphic disc contained in the $y$-axis, representing the relative class $-\alpha$ and with associated weight $z_{-\alpha}=w^{-1}$.

Since the projection $f$ is holomorphic, holomorphic discs of Maslov index 2 in $\left(\mathbb{C}^{2}, T_{r, \lambda}\right)$ are sections of $f$ over the disc of radius $r$ centered at $\epsilon$. When $r>|\epsilon|$ there are two families of such discs; these can be found either by explicit calculation, or by deforming $T_{r, \lambda}$ to a product torus $S^{1}\left(r_{1}\right) \times$ $S^{1}\left(r_{2}\right)$ (by deforming the circle centered at $\epsilon$ to a circle centered at the origin, without crossing $\epsilon$ ), for which the discs are simply $D^{2}\left(r_{1}\right) \times\{y\}$ and $\{x\} \times D^{2}\left(r_{2}\right)$. Denote by $\beta_{1}$ and $\beta_{2}$ respectively the classes of these discs, and by $z_{1}$ and $z_{2}$ the corresponding weights, which satisfy $z_{1} / z_{2}=$ $w$. In terms of these coordinates on $M$ the superpotential is then given by $W=z_{1}+z_{2}$.

On the other hand, when $r<|\epsilon|$ there is only one family of Maslov index 2 discs in $\left(\mathbb{C}^{2}, T_{r, \lambda}\right)$. This is easiest to see by deforming $T_{r, \lambda}$ to the Chekanov torus $|x y-\epsilon|=r,|x|=|y|$ (if $\omega$ is invariant under $x \leftrightarrow y$ this is simply $T_{r, 0}$ ); then the maximum principle applied to $y / x$ implies that Maslov index 2 discs are portions of lines $y=a x,|a|=1$. Denoting by $\beta_{0}$ the class of this disc, and by $u$ the corresponding weight, in the region $r<|\epsilon|$ the superpotential is $W=u$.

When we increase the value of $r$ past $r=|\epsilon|$, for $\lambda>0$, the family of holomorphic discs in the class $\beta_{0}$ deforms naturally into the family of discs in the class $\beta_{2}$ mentioned above; the coordinates on $M$ naturally glue according to $u=z_{2}, w=z_{1} / z_{2}$. On the other hand, for $\lambda<0$ the class $\beta_{0}$ deforms naturally into the class $\beta_{1}$, so that the coordinates glue according to $u=z_{1}$, $w=z_{1} / z_{2}$. The discrepancy in these gluings is due to the monodromy of our special Lagrangian fibration around the singular fiber $T_{|\epsilon|, 0}$, which acts nontrivially on $\pi_{2}\left(\mathbb{C}^{2}, T_{r, \lambda}\right)$ : while the coordinate $w$ is defined globally on $M, z_{1}$ and $z_{2}$ do not extend to global coordinates. 
There are now two issues: the complex manifold $M$ does not extend across the singularity at $(r, \lambda)=(|\epsilon|, 0)$, and the superpotential $W$ is discontinuous across the walls. Both issues are fixed simultaneously by instanton corrections. Namely, we correct the coordinate change across the wall $r=|\epsilon|, \lambda>0$ to $u=z_{2}(1+w)$. The correction factor $1+w$ indicates that, upon deforming $T_{r, \lambda}$ by increasing the value of $r$ past $|\epsilon|$, Maslov index 2 discs in the class $\beta_{0}$ give rise not only to discs in the class $\beta_{2}$ (by a straightforward deformation), but also to new discs in the class $\beta_{1}=\beta_{2}+\alpha$ formed by attaching the exceptional disc bounded by $T_{|\epsilon|, \lambda}$. Similarly, across $r=|\epsilon|$, $\lambda<0$, we correct the gluing to $u=z_{1}\left(1+w^{-1}\right)$, to take into account the exceptional disc in the class $-\alpha$ bounded by $T_{|\epsilon|, \lambda}$.

The corrected gluings both come out to be $u=z_{1}+z_{2}$, which means that we now have a well-defined mirror $X^{\vee}$, carrying a well-defined superpotential $W=u=z_{1}+z_{2}$. More precisely, using the coordinates $(u, w)$ on the chamber $r<|\epsilon|$, and the coordinates $(v, w)$ with $v=z_{2}^{-1}$ and $w=z_{1} / z_{2}$ on the chamber $r>|\epsilon|$, we claim that the corrected and completed mirror is

$$
X^{\vee}=\left\{(u, v, w) \in \mathbb{C}^{2} \times \mathbb{C}^{*}, u v=1+w\right\}, \quad W=u .
$$

More precisely, the region $r>|\epsilon|$ of our special Lagrangian fibration corresponds to $\left|z_{1}\right|$ and $\left|z_{2}\right|$ small, i.e. $|v|$ large; whereas the region $r<|\epsilon|$ corresponds to $|u|$ large compared to $e^{-|\epsilon|}$. When considering $M$ we also have $|u|<1$, as $|u| \rightarrow 1$ corresponds to $r \rightarrow 0$, but this constraint is removed by the completion process, which enlarges $X$ along the conic $x y=\epsilon$ by symplectic inflation. It turns out that we also have to complete $X^{\vee}$ in the "intermediate" region where $u$ and $v$ are both small, in particular allowing these variables to vanish; for otherwise, the corrected mirror would have "gaps" in the heavily corrected region near $(r, \lambda)=(|\epsilon|, 0)$. Let us also point out that $X^{\vee}$ is again the complement of a conic in $\mathbb{C}^{2}$.

General features of wall-crossing in Floer theory ensure that, when crossing a wall, holomorphic disc counts (and hence the superpotential) can be made to match by introducing a suitable analytic change of coordinates, consistently for all homotopy classes (see $\S 19.1$ of $[\mathbf{1 4}]$ and $\S 3$ of [4]). For instance, if we compactified $\mathbb{C}^{2}$ to $\mathbb{C P}^{2}$ or $\mathbb{C P}^{1} \times \mathbb{C P}^{1}$, then the tori $T_{r, \lambda}$ would bound additional families of Maslov index 2 holomorphic discs (passing through the divisors at infinity), leading to additional terms in the superpotential; however, these terms also match under the corrected gluing $u=z_{1}+z_{2}($ see $\S 5$ of $[4])$.

EXAMPLE 3.1.2. Consider $\mathbb{C}^{2}$ equipped with the standard holomorphic volume form $d \log x \wedge d \log y$ (with poles along the coordinate axes), and blow up the point $(1,0)$. This yields a complex manifold $X$ equipped with the holomorphic volume form $\Omega=\pi^{*}(d \log x \wedge d \log y)$, with poles along the proper transform $D$ of the coordinate axes. Observe that the $S^{1}$-action $e^{i \theta} \cdot(x, y)=$ $\left(x, e^{i \theta} y\right)$ lifts to $X$, and consider an $S^{1}$-invariant Kähler form $\omega$ for which the area of the exceptional divisor is $\epsilon$. Denote by $\mu_{S^{1}}: X \rightarrow \mathbb{R}$ the moment 
map for the $S^{1}$-action, normalized to equal 0 on the proper transform of the $x$-axis and $\epsilon$ at the isolated fixed point. Then the $S^{1}$-invariant tori

$$
L_{r, \lambda}=\left\{\left|\pi^{*} x\right|=r, \mu_{S^{1}}=\lambda\right\}
$$

define a special Lagrangian fibration on $X \backslash D$, with a nodal singularity at the isolated fixed point (for $(r, \lambda)=(1, \epsilon)$ ) [2].

The base of this special Lagrangian fibration is pictured on Figure 2, where the vertical axis corresponds to the moment map, and a cut has been made below the singular point to depict the monodromy of the symplectic affine structure.

For $r=1$ the Lagrangian tori $L_{r, \lambda}$ bound exceptional holomorphic discs, which causes wall-crossing: for $\lambda>\epsilon, L_{1, \lambda}$ bounds a Maslov index 0 disc in the proper transform of the line $x=1$, whereas for $\lambda<\epsilon, L_{1, \lambda}$ splits the exceptional divisor of the blowup into two discs, one of which has Maslov index 0 . Thus, we have to consider the chambers $r>1$ and $r<1$ separately.

When $r<1$, the Lagrangian torus $L_{r, \lambda}$ bounds two families of Maslov index 2 discs. One family consists of the portions where $\mu_{S^{1}}<\lambda$ of the lines $x=$ constant; we denote by $\delta$ the homotopy class of these discs, and by $z\left(=z_{\delta}\right)$ the corresponding holomorphic coordinate on $M$, which satisfies $|z|=e^{-\lambda}$. The other family consists of discs intersecting the $y$-axis, and is easiest to see by deforming $L_{r, \lambda}$ to a product torus, upon which it becomes the family of discs of radius $r$ in the lines $y=$ constant. (In fact, $L_{r, \lambda}$ is typically already a product torus for $r$ sufficiently different from 1 , when it lies in the region where the blow-up operation does not affect the Kähler form.) We denote by $\beta$ the class of these discs, and by $u$ the corresponding holomorphic coordinate on $M$. The coordinates $u$ and $z$ on $M$ can be thought of as (exponentiated) complexifications of the affine coordinates on the base pictured on Figure 2 .

On the other hand, when $r>1$ the torus $L_{r, \lambda}$ bounds three families of Maslov index 2 discs. As before, one of these families consists of the portions where $\mu_{S^{1}}<\lambda$ of the lines $x=$ constant, contributing $z=z_{\delta}$ to

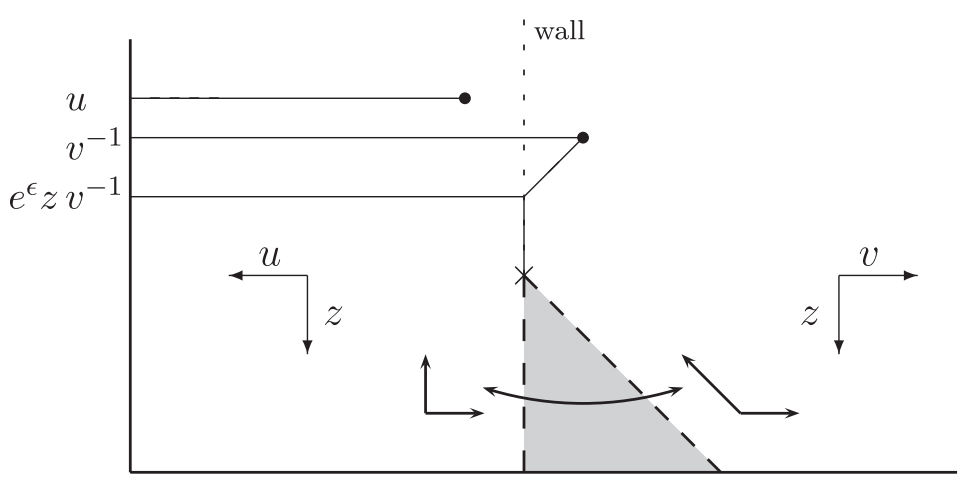

FiguRE 2. A special Lagrangian fibration on the blowup of $\mathbb{C}^{2}$ 
the superpotential. The two other families intersect the $y$-axis, and can be described explicitly when $L_{r, \lambda}$ is a product torus (away from the blown up region): one consists as before of discs of radius $r$ in the lines $y=$ constant, while the other one consists of the proper transforms of discs which hit the $x$-axis at $(1,0)$, namely the family of discs $z \mapsto(r z, \rho(r z-1) /(r-z))$ for fixed $|\rho|$. Denote by $v$ the complexification of the right-pointing affine coordinate on Figure 2 in the chamber $r>1$, normalized so that, if we ignore instanton corrections, the gluing across the wall $(r=1, \lambda>\epsilon)$ is given by $u=v^{-1}$. Then the two families of discs intersecting the $y$-axis contribute respectively $v^{-1}$ and $e^{\epsilon} z v^{-1}$ to the superpotential; the first family survives the wall-crossing at $r=1$, while the second one degenerates by bubbling of an exceptional disc (the part of the proper transform of the line $x=1$ where $\mu_{S^{1}}<\lambda$ ). This phenomenon is pictured on Figure 2 (where the various discs are abusively represented as tropical curves, which actually should be drawn in the complex affine structure).

Thus the instanton-corrected gluing is given by $u=v^{-1}+e^{\epsilon} z v^{-1}$ across the wall $(r=1, \lambda>\epsilon)$; and a similar analysis shows that the portion of the wall where $\lambda<\epsilon$ also gives rise to the same instanton-corrected gluing. Thus, the instanton-corrected and completed mirror is given by

$$
X^{\vee}=\left\{(u, v, z) \in \mathbb{C}^{2} \times \mathbb{C}^{*}, u v=1+e^{\epsilon} z\right\}, \quad W=u+z .
$$

(Before completing the mirror by symplectically enlarging $X$, we would impose the restrictions $|u|<1$ and $|z|<1$.) The reader is referred to [2] for more details.

Remark. The above examples are particularly simple, as they involve a single singularity of the special Lagrangian fibration and a single wall-crossing correction. In more complicated examples, additional walls are generated by intersections between the "primary" walls emanating from the singularities; in the end there are infinitely many walls, and hence infinitely many instanton corrections to take into account when constructing the mirror. A framework for dealing with such situations has been introduced by Kontsevich and Soibelman [29], see also the work of Gross and Siebert [18, 19].

3.2. Beyond the Fano case: Hirzebruch surfaces. The construction of the mirror superpotential for toric Fano varieties is well-understood (see e.g. $[\mathbf{2 1}, \mathbf{1 1}, \mathbf{4}, \mathbf{1 5}]$ for geometric derivations), and has been briefly summarized in $\S 2.3$ above. As pointed out to the author by Kenji Fukaya, in the non-Fano case the superpotential differs from the formula in Proposition 2.5 by the presence of additional terms, which count the virtual contributions of Maslov index 2 configurations consisting of a disc of Maslov index 2 or more together with a collection of spheres of non-positive Chern number. A non-explicit formula describing the general shape of the additional terms has been given by Fukaya-Oh-Ohta-Ono: compare Theorems 3.4 and 3.5 in 
[15]. In this section we derive an explicit formula for the full superpotential in the simplest example, using wall-crossing calculations.

The simplest non-Fano toric examples are rational ruled surfaces, namely the Hirzebruch surfaces $\mathbb{F}_{n}=\mathbb{P}\left(\mathcal{O}_{\mathbb{P}^{1}} \oplus \mathcal{O}_{\mathbb{P}^{1}}(n)\right)$ for $n \geq 2$. The mirror of $\mathbb{F}_{n}$ is still $\left(\mathbb{C}^{*}\right)^{2}$, but with a superpotential of the form $W=W_{0}+$ additional terms $[\mathbf{1 5}]$, where $W_{0}$ is given by (2.5), namely in this case

$$
W_{0}(x, y)=x+y+\frac{e^{-[\omega] \cdot\left[S_{n}\right]}}{x y^{n}}+\frac{e^{-[\omega] \cdot[F]}}{y},
$$

where $[F] \in H_{2}\left(\mathbb{F}_{n}\right)$ is class of the fiber, and $\left[S_{n}\right]$ is the class of a section of square $n$. The superpotential $W_{0}$ has $n+2$ critical points, four of which lie within the region of $\left(\mathbb{C}^{*}\right)^{2}$ which maps to the moment polytope via the logarithm map. Discarding the other critical points (i.e., restricting to the appropriate subset of $\left.\left(\mathbb{C}^{*}\right)^{2}\right)$, homological mirror symmetry can be shown to hold for (a deformation of) $W_{0}[\mathbf{5}]$ (see also [1]). However, this is unsatisfactory for various reasons, among others the discrepancy between the critical values of $W_{0}$ and the eigenvalues of quantum cup-product with the first Chern class in $Q H^{*}\left(\mathbb{F}_{n}\right)$ (see e.g. $\S 6$ of $[\mathbf{4}]$, and [15]).

The approach we use to compute the full superpotential relies on the observation that, depending on the parity of $n, \mathbb{F}_{n}$ is deformation equivalent, and in fact symplectomorphic, to either $\mathbb{F}_{0}=\mathbb{C P}^{1} \times \mathbb{C P}^{1}$ or $\mathbb{F}_{1}$ (the one-point blowup of $\mathbb{C P}^{2}$ ), equipped with a suitable symplectic form. Carrying out the deformation explicitly provides a way of achieving transversality for the Floer theory of Lagrangian tori in $\mathbb{F}_{n}$, by deforming the non-regular complex structure of $\mathbb{F}_{n}$ to a regular one. In the case of $\mathbb{F}_{2}$ and $\mathbb{F}_{3}$ at least, the result of the deformation can be explicitly matched with $\mathbb{F}_{0}$ or $\mathbb{F}_{1}$ equipped with a non-toric holomorphic volume form of the type considered in Example 3.1.1, which allows us to compute the superpotential $W$. In the case of $\mathbb{F}_{2}$ the deformation we want to carry out is pictured schematically in Figure 3.

Proposition 3.1. The corrected superpotential on the mirror of $\mathbb{F}_{2}$ is the Laurent polynomial

$$
W(x, y)=x+y+\frac{e^{-[\omega] \cdot\left[S_{+2}\right]}}{x y^{2}}+\frac{e^{-[\omega] \cdot[F]}}{y}+\frac{e^{-[\omega] \cdot\left[S_{-2}\right]} e^{-[\omega] \cdot[F]}}{y} .
$$

This formula differs from $W_{0}$ by the addition of the last term; geometrically, this term corresponds to configurations consisting of a Maslov index 2

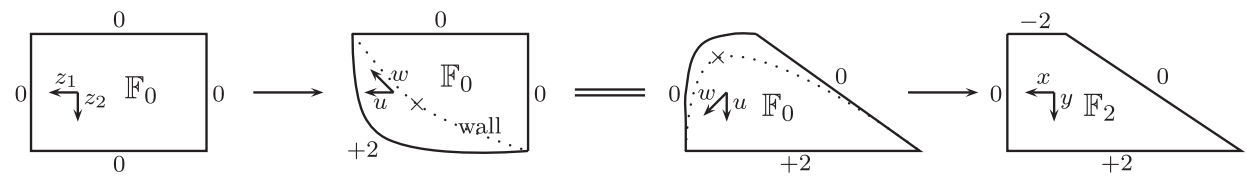

FiguRe 3 . Deforming $\mathbb{F}_{0}$ to $\mathbb{F}_{2}$ 
disc intersecting the exceptional section $S_{-2}$ together with the exceptional section itself.

Proof. Consider the family of quadric surfaces $\mathcal{X}=\left\{x_{0} x_{1}=x_{2}^{2}-\right.$ $\left.t^{2} x_{3}^{2}\right\} \subset \mathbb{C P}^{3} \times \mathbb{C}$. For $t \neq 0, X_{t}=\left\{x_{0} x_{1}=\left(x_{2}+t x_{3}\right)\left(x_{2}-t x_{3}\right)\right\} \subset \mathbb{C P}^{3}$ is a smooth quadric, and can be explicitly identified with the image of the embedding of $\mathbb{C P}^{1} \times \mathbb{C P}^{1}$ given in homogeneous coordinates by

$$
i_{t}:\left(\left(\xi_{0}: \xi_{1}\right),\left(\eta_{0}: \eta_{1}\right)\right) \mapsto\left(\xi_{0} \eta_{1}: \xi_{1} \eta_{0}: \frac{1}{2}\left(\xi_{0} \eta_{0}+\xi_{1} \eta_{1}\right): \frac{1}{2 t}\left(\xi_{0} \eta_{0}-\xi_{1} \eta_{1}\right)\right),
$$

or, in terms of the affine coordinates $x=\xi_{0} / \xi_{1}$ and $y=\eta_{0} / \eta_{1}$,

$$
i_{t}(x, y)=\left(x: y: \frac{1}{2}(x y+1): \frac{1}{2 t}(x y-1)\right) .
$$

For $t=0$, the surface $X_{0}=\left\{x_{0} x_{1}=x_{2}^{2}\right\}$ is a cone with vertex at the point $(0: 0: 0: 1)$, where the 3 -fold $\mathcal{X}$ itself presents an ordinary double point singularity. Denote by $\pi: \mathcal{X}^{\prime} \rightarrow \mathcal{X}$ a small resolution: composing with the projection to $\mathbb{C}$, we obtain a family of surfaces $X_{t}^{\prime}$, such that $X_{t}^{\prime} \cong X_{t}$ for $t \neq 0$, while $X_{0}^{\prime}$ is the blowup of $X_{0}$, namely $X_{0}^{\prime} \cong \mathbb{F}_{2}$.

Consider the family of anticanonical divisors

$$
D_{t}=\left\{x_{0}=0, x_{2}=t x_{3}\right\} \cup\left\{x_{1}=0, x_{2}=t x_{3}\right\} \cup\left\{x_{3}=-t x_{2}\right\} \subset X_{t},
$$

and equip $X_{t}$ with a holomorphic volume form $\Omega_{t}$ with poles along $D_{t}$. Observe that $D_{t}$ is the image by $i_{t}$ of the lines at infinity $\xi_{1}=0$ and $\eta_{1}=0$, and of the conic $C_{t}: \xi_{0} \eta_{0}=\frac{1-t^{2}}{1+t^{2}} \xi_{1} \eta_{1}$, i.e. in affine coordinates, $x y=\frac{1-t^{2}}{1+t^{2}}$. Thus, for $t=1$ the pair $\left(X_{t}, D_{t}\right)$ corresponds to the toric anticanonical divisor in $\mathbb{C P}^{1} \times \mathbb{C P}^{1}$, while for general $t$ the geometry of $\left(X_{t}, D_{t}, \Omega_{t}\right)$ resembles closely that of Example 3.1.1 (the only difference is that we have compactified $\mathbb{C}^{2}$ to $\left.\mathbb{C P}^{1} \times \mathbb{C P}^{1}\right)$. Finally, $D_{0} \subset X_{0}$ is precisely the toric anticanonical divisor, consisting of the lines $x_{0}=0, x_{1}=0$ (two rays of the cone) and the conic $x_{3}=0$ (the base of the cone).

The quadrics $X_{t}$, the divisors $D_{t}$ and the volume forms $\Omega_{t}$ are preserved by the $S^{1}$-action $\left(x_{0}: x_{1}: x_{2}: x_{3}\right) \mapsto\left(x_{0} e^{i \theta}: x_{1} e^{-i \theta}: x_{2}: x_{3}\right)$, and so is the Kähler form induced by restriction of the Fubini-Study Kähler form on $\mathbb{C P}^{3}$. Moreover, for $t=1$ the standard $T^{2}$-action on $\mathbb{C P}^{1} \times \mathbb{C P}^{1}$ is induced by a subgroup of $P U(4)$, so that the Kähler form is toric, and the configuration at $t=0$ is also toric (with respect to a different $T^{2}$-action!); however for general $t$ we only have $S^{1}$-invariance.

The $S^{1}$-action lifts to the small resolution, and the lifted divisors $D_{t}^{\prime}=$ $\pi^{-1}\left(D_{t}\right) \subset X_{t}^{\prime}$ and holomorphic volume forms $\Omega_{t}^{\prime}=\pi^{*} \Omega_{t}$ are $S^{1}$-invariant. Additionally, $\mathcal{X}^{\prime}$ can be equipped with a $S^{1}$-invariant Kähler form, whose cohomology class depends on the choice of a parameter (the symplectic area of the exceptional -2-curve); restricting to $X_{t}^{\prime}$, we obtain a family of $S^{1}$-invariant Kähler forms $\omega_{t}^{\prime}$. Moreover, careful choices can be made in the construction in order to ensure that the Kähler forms $\omega_{0}^{\prime}$ and $\omega_{1}^{\prime}$ on $X_{0}^{\prime}$ and $X_{1}^{\prime}$ are invariant under the respective $T^{2}$-actions. 
From the symplectic point of view, the family $\left(X_{t}^{\prime}, \omega_{t}^{\prime}\right)$ is trivial; however, the complex structure for $t=0$ is non-generic. At $t=1$ and $t=0$ the anticanonical divisors are the toric ones for $\mathbb{F}_{0}$ and $\mathbb{F}_{2}$ respectively; deforming away from these values, we partially smooth the toric anticanonical divisor, which in the case of $\mathbb{F}_{2}$ requires a simultaneous deformation of the complex structure because the exceptional curve $S_{-2}$ is rigid. The deformation from $t=1$ to $t=0$ is now as pictured on Figure 3, which the reader is encouraged to keep in mind for the rest of the argument.

For $t=1$, the mirror superpotential is given by the formula for the toric Fano case (2.5), namely

$$
W=z_{1}+z_{2}+e^{-A} z_{1}^{-1}+e^{-B} z_{2}^{-1},
$$

where $A$ and $B$ are the symplectic area of the two $\mathbb{C P}^{1}$ factors; the first two terms correspond to discs contained in the affine chart with coordinates $x$ and $y$ we have considered above, while the last two terms correspond to discs which hit the lines at infinity. Deforming to general $t$, we have $X_{t}^{\prime} \backslash D_{t}^{\prime} \cong \mathbb{C}^{2} \backslash\left\{x y=\frac{1-t^{2}}{1+t^{2}}\right\}$. Even though the Kähler form $\omega_{t}^{\prime}$ is not toric, the construction of an $S^{1}$-invariant special Lagrangian fibration proceeds exactly as in Example 3.1.1. The discussion carries over with only one modification: when considered as submanifolds of $\mathbb{C P}^{1} \times \mathbb{C P}^{1}$, the tori $T_{r, \lambda}=\left\{\left|x y-\frac{1-t^{2}}{1+t^{2}}\right|=r, \mu_{S^{1}}=\lambda\right\}$ bound additional families of Maslov index 2 holomorphic discs (intersecting the lines at infinity).

In the chamber $r>\left|\frac{1-t^{2}}{1+t^{2}}\right|$, deforming $T_{r, \lambda}$ to a product torus shows that it bounds four families of Maslov index 2 discs, and the superpotential is given by (3.3) as in the toric case. On the other hand, in the chamber $r<\left|\frac{1-t^{2}}{1+t^{2}}\right|$, deforming $T_{r, \lambda}$ to the Chekanov torus shows that it bounds five families of Maslov index 2 holomorphic discs; explicit calculations are given in Section 5.4 of $[4]$. (In [4] it was assumed for simplicity that the two $\mathbb{C P}^{1}$ factors had equal symplectic areas, but it is easy to check that the discussion carries over to the general case without modification.) Using the same notations as in Example 3.1.1, the superpotential is now given by

$$
W=u+\frac{e^{-A}(1+w)}{u w}+\frac{e^{-B}(1+w)}{u}
$$

(see Corollary 5.13 in [4]). The first term $u$ corresponds to the family of discs which are sections of $f:(x, y) \mapsto x y$ over the disc $\Delta$ of radius $r$ centered at $\frac{1-t^{2}}{1+t^{2}}$; these discs pass through the conic $x y=\left(1-t^{2}\right) /\left(1+t^{2}\right)$ and avoid all the toric divisors. The other terms correspond to sections of $f$ over $\mathbb{C P}^{1} \backslash \Delta$. These discs intersect exactly one of the two lines at infinity, and one of the two coordinate axes; each of the four possibilities gives rise to one family of holomorphic discs. The various cases are as follows (see Proposition 5.12 in $[4])$ : 


\begin{tabular}{|c||c|c|c|c||c|}
\hline class & $x=0$ & $y=0$ & $x=\infty$ & $y=\infty$ & weight \\
\hline$H_{1}-\beta_{0}-\alpha$ & no & yes & yes & no & $e^{-A} / u w$ \\
$H_{1}-\beta_{0}$ & yes & no & yes & no & $e^{-A} / u$ \\
$H_{2}-\beta_{0}$ & no & yes & no & yes & $e^{-B} / u$ \\
$H_{2}-\beta_{0}+\alpha$ & yes & no & no & yes & $e^{-B} w / u$ \\
\hline
\end{tabular}

Here $H_{1}=\left[\mathbb{C P}^{1} \times\{p t\}\right], H_{2}=\left[\{p t\} \times \mathbb{C P}^{1}\right]$, and $\beta_{0}$ and $\alpha$ are the classes in $\pi_{2}\left(\mathbb{C}^{2}, T_{r, \lambda}\right)$ introduced in Example 3.1.1.

Here as in Example 3.1.1, it is easy to check that the two formulas for the superpotential are related by the change of variables $w=z_{1} / z_{2}$ and $u=$ $z_{1}+z_{2}$, which gives the instanton-corrected gluing between the two chambers.

The tori $T_{r, \lambda}$ with $r<\left|\frac{1-t^{2}}{1+t^{2}}\right|$ cover the portion of $X_{t}^{\prime} \backslash D_{t}^{\prime}$ where $\left|x y-\frac{1-t^{2}}{1+t^{2}}\right|<\left|\frac{1-t^{2}}{1+t^{2}}\right|$, which under the embedding $i_{t}$ corresponds to the inequality

$$
\left|x_{2}-t x_{3}\right|>\left|\frac{2 t}{1-t^{2}}\right|\left|t x_{2}+x_{3}\right|
$$

For $t \rightarrow 0$ this region covers almost all of $X_{t}^{\prime} \backslash D_{t}^{\prime}$, with the exception of a small neighborhood of the lines $\left\{x_{0}=0, x_{2}=t x_{3}\right\}$ and $\left\{x_{1}=0, x_{2}=t x_{3}\right\}$. On the other hand, as $t \rightarrow 0$ the family of special Lagrangian tori $T_{r, \lambda}$ converge to the standard toric Lagrangian fibration on $X_{0}^{\prime}=\mathbb{F}_{2}$, without any further wall-crossing as $t$ approaches zero provided that $r$ is small enough for $T_{r, \lambda}$ to lie within the correct chamber. It follows that, in suitable coordinates, the superpotential for the Landau-Ginzburg mirror to $\mathbb{F}_{2}$ is given by (3.4).

All that remains to be done is to express the coordinates $x$ and $y$ in (3.2) in terms of $u$ and $w$. In order to do this, we investigate the limiting behaviors of the five families of discs contributing to (3.4) as $t \rightarrow 0$ : four of these families are expected to converge to the "standard" families of Maslov index 2 discs in $\mathbb{F}_{2}$, since those are all regular. Matching the families of discs allows us to match four of the terms in (3.4) with the four terms in $W_{0}$. The leftover term in (3.4) will then correspond to the additional term in (3.2).

Consider a family of tori $T_{r(t), \lambda(t)}$ in $X_{t}^{\prime}$ which converge to a $T^{2}$-orbit in $X_{0}^{\prime}=\mathbb{F}_{2}$, corresponding to fixed ratios $\left|x_{0}\right| /\left|x_{3}\right|=\rho_{0}$ and $\left|x_{1}\right| /\left|x_{3}\right|=\rho_{1}$ (and hence $\left.\left|x_{2}\right| /\left|x_{3}\right|=\sqrt{\rho_{0} \rho_{1}}\right)$. Since the small resolution $\pi: \mathcal{X}^{\prime} \rightarrow \mathcal{X}$ is an isomorphism away from the exceptional curve in $X_{0}^{\prime}$, we can just work on $\mathcal{X}$ and use the embeddings $i_{t}$ to convert back and forth between coordinates on $X_{t}^{\prime} \cong X_{t} \cong \mathbb{C P}^{1} \times \mathbb{C P}^{1}$ and homogeneous coordinates in $\mathbb{C P}^{3}$ for $t \neq 0$. Since

$$
\frac{\left|x_{2}\right|}{\left|x_{3}\right|}=\left|t \frac{x y+1}{x y-1}\right|=\left|t+\frac{2 t}{x y-1}\right|
$$

should converge to a finite non-zero value as $t \rightarrow 0$, the value of $|x y-1|$ must converge to zero, and hence $r(t)=\left|x y-\frac{1-t^{2}}{1+t^{2}}\right|$ must also converge to 0 ; in fact, an easily calculation shows that $r(t) \sim 2|t| / \sqrt{\rho_{0} \rho_{1}}$. Therefore, 
for $t$ small, $x y$ is close to 1 everywhere on $T_{r(t), \lambda(t)}$. On the other hand, $|x| /|y|=\left|x_{0}\right| /\left|x_{1}\right|$ converges to the finite value $\rho_{0} / \rho_{1}$; thus $|x|$ and $|y|$ are bounded above and below on $T_{r(t), \lambda(t)}$. Now, consider a holomorphic disc with boundary in $T_{r(t), \lambda(t)}$, representing the class $H_{1}-\beta_{0}$. Since the $y$ coordinate has neither zeroes nor poles, by the maximum principle its norm is bounded above and below by fixed constants (independently of $t$ ). The point where the disc hits the line $x=\infty$ (i.e., $\left.\xi_{1}=0\right)$ has coordinates $\left(x_{0}: x_{1}: x_{2}: x_{3}\right)=$ $\left(\xi_{0} \eta_{1}: 0: \frac{1}{2} \xi_{0} \eta_{0}: \frac{1}{2 t} \xi_{0} \eta_{0}\right)=(1: 0: y: y / 2 t)$, which given the bounds on $|y|$ converges to the singular point $(0: 0: 0: 1)$ as $t \rightarrow 0$. Thus, as $t \rightarrow 0$, this family of discs converges to stable maps in $X_{0}^{\prime}$ which have non-empty intersection with the exceptional curve. The same argument (exchanging $x$ and $y$ ) also applies to the discs in the class $H_{2}-\beta_{0}$. On the other hand, the three other families of discs can be shown to stay away from the exceptional curve.

In $\mathbb{F}_{2}$, the $T^{2}$-orbits bound four regular families of Maslov index 2 holomorphic discs, one for each component of the toric anticanonical divisor $D_{0}^{\prime}=S_{+2} \cup S_{-2} \cup F_{0} \cup F_{1}$; here $S_{-2}$ is the exceptional curve, $S_{+2}$ is the preimage by $\pi$ of the component $\left\{x_{3}=0\right\}$ of $D_{0} \subset X_{0}$, and $F_{0}$ and $F_{1}$ are two fibers of the ruling, namely the proper transforms under $\pi$ of the lines $x_{0}=0$ and $x_{1}=0$ in $X_{0}$. The four families of discs can be constructed explicitly in coordinates as in the proof of Proposition 2.5, see eq. (2.6); regularity implies that, as we deform $X_{0}^{\prime}=\mathbb{F}_{2}$ to $X_{t}^{\prime}$ for $t \neq 0$ small enough, all these discs deform to holomorphic discs in $\left(X_{t}^{\prime}, T_{r(t), \lambda(t)}\right)$.

The term $y$ in $W_{0}$ corresponds to the family of discs intersecting the section $S_{+2}$, which under the projection $X_{0}^{\prime} \rightarrow X_{0}$ corresponds to the component $\left\{x_{3}=0\right\}$ of the divisor $\left.D_{0}\right)$. Thus, its deformation for $t \neq 0$ intersects the component $\left\{x_{3}=-t x_{2}\right\}$ of the divisor $D_{t}$, namely the conic $C_{t}$ in the affine part of $\mathbb{C P}^{1} \times \mathbb{C P}^{1}$. Comparing the contributions to the superpotential, we conclude that $y=u$.

Next, the term $x$ in $W_{0}$ corresponds to the family of discs intersecting the ruling fiber $F_{0}$, which projects to the line $\left\{x_{0}=0\right\}$ on $X_{0}$. Thus, for small enough $t \neq 0$ these discs deform to a family of discs in $X_{t}^{\prime}$ that intersect the component $\left\{x_{0}=0, x_{2}=t x_{3}\right\}$ of $D_{t}^{\prime}$, i.e. the line at infinity $\eta_{1}=0$. There are two such families, in the classes $H_{2}-\beta_{0}$ and $H_{2}-\beta_{0}+\alpha$; however we have seen that the discs in the class $H_{2}-\beta_{0}$ approach the exceptional curve as $t \rightarrow 0$, which would give a contradiction. Thus the term $x$ in $W_{0}$ corresponds to the family of discs in the class $H_{2}-\beta_{0}+\alpha$, which gives $x=e^{-B} w / u$.

The proof is then completed by observing that the change of variables $x=e^{-B} w / u, y=u$ identifies (3.2) with (3.4). (Recall that the symplectic areas of $S_{+2}, S_{-2}$, and the ruling fibers in $\mathbb{F}_{2}$ are respectively $A+B, A-B$, and $B)$.

Note: as a quick consistency check, our change of variables matches the term $e^{-(A+B)} / x y^{2}$ in $(3.2)$, which corresponds to discs in $\mathbb{F}_{2}$ that intersect the ruling fiber $F_{1}$, with the term $e^{-A} / u w$ in (3.4), which corresponds to discs representing the class $H_{1}-\beta_{0}-\alpha$ in $X_{t}^{\prime}$ and intersecting the line at infinity $\xi_{1}=0$. The remaining two terms in (3.4) correspond to discs representing 
the classes $H_{1}-\beta_{0}$ and $H_{2}-\beta_{0}$ in $X_{t}^{\prime}$, whose limits as $t \rightarrow 0$ intersect the exceptional curve $S_{-2}$, and can also be matched to the remaining terms in (3.2).

A similar method can be applied to the case of $\mathbb{F}_{3}$, and yields:

Proposition 3.2. The corrected superpotential on the mirror of $\mathbb{F}_{3}$ is the Laurent polynomial

$W(x, y)=x+y+\frac{e^{-[\omega] \cdot\left[S_{+3}\right]}}{x y^{3}}+\frac{e^{-[\omega] \cdot[F]}}{y}+\frac{2 e^{-[\omega] \cdot\left(\left[S_{-3}\right]+2[F]\right)}}{y^{2}}+\frac{e^{-[\omega] \cdot\left(\left[S_{-3}\right]+[F]\right)} x}{y}$.

Sketch OF PROOF. The deformation we want to carry out is now depicted on Figure 4. One way of constructing this deformation is to start with the family $\mathcal{X}^{\prime}$ considered previously, and perform a birational transformation. Namely, let $C^{\prime} \subset \mathcal{X}^{\prime}$ be the proper transform of the curve $C=$ $\left\{x_{0}=x_{1}=0, x_{2}=t x_{3}\right\} \subset \mathcal{X}$, and let $\hat{\mathcal{X}}^{\prime}$ be the blowup of $\mathcal{X}^{\prime}$ along $C^{\prime}$. (This amounts to blowing up the point $x=y=\infty$ in each quadric $X_{t}^{\prime}$ for $t \neq 0$, and the point where $S_{-2}$ intersects the fiber $F_{1}$ in $\left.X_{0}^{\prime} \cong \mathbb{F}_{2}\right)$. Next, let $\hat{Z} \subset \hat{\mathcal{X}}^{\prime}$ be the proper transform of the surface $Z=\left\{x_{1}=0, x_{2}=t x_{3}\right\} \subset \mathcal{X}$. Denote by $\mathcal{X}^{\prime \prime}$ the 3 -fold obtained by contracting $\hat{Z}$ in $\hat{\mathcal{X}}^{\prime}$ : namely, $\mathcal{X}^{\prime \prime}$ is a family of surfaces $X_{t}^{\prime \prime}$, each obtained from $X_{t}^{\prime}$ by first blowing up a point as explained above and then blowing down the proper transform of the line $x_{1}=0, x_{2}=t x_{3}$ (for $t \neq 0$ this is the line at infinity $\xi_{1}=0$, while for $t=0$ this is the ruling fiber $F_{1}$ ). One easily checks that $X_{t}^{\prime \prime} \cong \mathbb{F}_{1}$ for $t \neq 0$, while $X_{0}^{\prime \prime} \cong \mathbb{F}_{3}$. Moreover, the divisors $D_{t}^{\prime} \subset X_{t}^{\prime}$ transform naturally under the birational transformations described above, and yield a family of anticanonical divisors $D_{t}^{\prime \prime} \subset X_{t}^{\prime \prime}$; for $t=0$ and $t=1$ these are precisely the toric anticanonical divisors in $X_{0}^{\prime \prime}=\mathbb{F}_{3}$ and $X_{1}^{\prime \prime}=\mathbb{F}_{1}$.

In terms of the affine charts on $X_{t}^{\prime} \cong \mathbb{F}_{0}$ considered in the proof of Proposition 3.1, the birational transformations leading to $\left(X_{t}^{\prime \prime}, D_{t}^{\prime \prime}\right)$ are performed "at infinity": thus $D_{t}^{\prime \prime}$ is again the union of the conic $x y=\frac{1-t^{2}}{1+t^{2}}$ and the divisors at infinity, namely for general $t$ we are again dealing with a compactified version of Example 3.1.1. Thus $X_{t}^{\prime \prime} \backslash D_{t}^{\prime \prime}$ still contains an $S^{1}$-invariant family of special Lagrangian tori $T_{r, \lambda}$, constructed as previously, and there are again two chambers separated by the wall $r=\left|\frac{1-t^{2}}{1+t^{2}}\right|$; the only difference

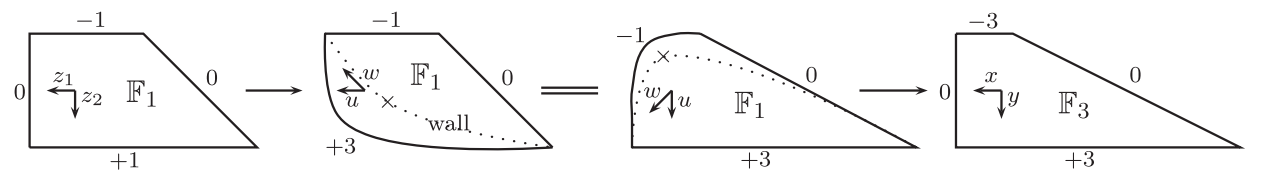

Figure 4. Deforming $\mathbb{F}_{1}$ to $\mathbb{F}_{3}$ 
concerns the superpotential, since the tori $T_{r, \lambda}$ now bound different families of holomorphic discs passing through the divisors at infinity. These families and their contributions to the superpotential can be determined by the same techniques as in the cases of $\mathbb{C P}^{2}$ and $\mathbb{C P}^{1} \times \mathbb{C P}^{1}$, which are treated in Section 5 of [4]. Namely, for $r>\left|\frac{1-t^{2}}{1+t^{2}}\right|$ the tori $T_{r, \lambda}$ can be isotoped to product tori, and hence they bound four families of Maslov index 2 discs, giving the familiar formula

$$
W=z_{1}+z_{2}+\frac{e^{-(A+B)}}{z_{1} z_{2}}+\frac{e^{-B}}{z_{2}}
$$

where $B$ is the area of the ruling fiber in $\mathbb{F}_{1}$ and $A$ is the area of the exceptional curve. Meanwhile, for $r<\left|\frac{1-t^{2}}{1+t^{2}}\right|$ the tori $T_{r, \lambda}$ can be isotoped to Chekanov tori; it can be shown that they bound 6 families of Maslov index 2 discs, and using the same notations as in Example 3.1.1 we now have

$$
W=u+\frac{e^{-(A+B)}(1+w)^{2}}{u^{2} w}+\frac{e^{-B}(1+w)}{u} .
$$

The instanton-corrected gluing between the two chambers is again given by $u=z_{1}+z_{2}$ and $w=z_{1} / z_{2}$; in fact (3.7) can be derived from (3.6) via this change of variables without having to explicitly determine the holomorphic discs bounded by $T_{r, \lambda}$.

The strategy is now the same as in the proof of Proposition 3.1: as $t \rightarrow 0$, the special Lagrangian fibrations on $X_{t}^{\prime \prime} \backslash D_{t}^{\prime \prime}$ converge to the standard fibration by $T^{2}$-orbits on $X_{0}^{\prime \prime} \simeq \mathbb{F}_{3}$, and the chamber $r<\left|\frac{1-t^{2}}{1+t^{2}}\right|$ covers arbitrarily large subsets of $X_{t}^{\prime \prime} \backslash D_{t}^{\prime \prime}$. Therefore, as before the superpotential for the Landau-Ginzburg mirror to $\mathbb{F}_{3}$ is given by (3.7) in suitable coordinates; the expression for the variables $x$ and $y$ in (3.5) in terms of $u$ and $w$ can be found by matching some of the families of discs bounded by $T_{r, \lambda}$ as $t \rightarrow 0$ to the regular families of Maslov index 2 discs bounded by the $T^{2}$-orbits in $\mathbb{F}_{3}$.

Concretely, the term $y$ in (3.1) corresponds to holomorphic discs in $\mathbb{F}_{3}$ which intersect the section $S_{+3}$. By regularity, these discs survive the deformation to a small nonzero value of $t$, and there they correspond to a family of discs which are entirely contained in the affine charts. Hence, as before we must have $y=u$. Identifying which term of (3.7) corresponds to the term $x$ in (3.1) requires more work, but can be done exactly along the same lines as for Proposition 3.1; in fact, we find that it is given by exactly the same formula $x=e^{-B} w / u$ as in the case of $\mathbb{F}_{2}$. A posteriori this is not at all surprising, since this family of discs stays away from the line at infinity $\xi_{1}=0$ in $X_{t}^{\prime}$, and hence lies in the part of $X_{t}^{\prime}$ that is not affected by the birational transformations that lead to $X_{t}^{\prime \prime}$.

Applying the change of variables $x=e^{-B} w / u, y=u$ to (3.7), and recalling that the symplectic areas of $S_{+3}, S_{-3}$ and the ruling fibers in $\mathbb{F}_{3}$ are respectively $A+2 B, A-B$, and $B$, we arrive at (3.5), which completes the proof. 
It is tempting to interpret the last two terms in (3.5) as the contributions of Maslov index 2 stable configurations that include the exceptional curve $S_{-3}$ as a bubble component. Namely, the next-to-last term should be a virtual count of configurations that consist of a double cover of a Maslov index 2 disc passing through $S_{-3}$, together with $S_{-3}$; and the last term should be a virtual count of configurations consisting of a Maslov index 4 disc which intersects both the ruling fiber $F_{0}$ and the exceptional section $S_{-3}$, together with $S_{-3}$.

In general, Fukaya-Oh-Ohta-Ono show that the "naive" superpotential $W_{0}$ should be corrected by virtual contributions of Maslov index 2 configurations for which transversality fails in the toric setting; moreover, they show that the perturbation data needed to make sense of the virtual counts can be chosen in a $T^{2}$-equivariant manner [15]. In principle, different choices of perturbation data could lead to different virtual counts of holomorphic discs, and hence to different formulas for the corrected superpotential. Our approach here can be understood as an explicit construction of a perturbation that achieves transversality for holomorphic discs, by deforming the complex structure to a generic one. However, our perturbation is only $S^{1}$-equivariant rather than $T^{2}$-equivariant, so it is not clear that our count of discs agrees with the virtual counts obtained by using Fukaya-Oh-OhtaOno's perturbation data (the latter have not been computed yet, in fact their direct computation seems extremely difficult). It is nonetheless our hope that the two counts might agree; from this perspective it is encouraging to note that open Gromov-Witten invariants are well-defined in the $S^{1}$-equivariant setting, and not just in the toric setting [31].

3.3. Higher dimensions. In this section we give two explicit local models for singularities of Lagrangian fibrations in higher dimensions and their instanton-corrected mirrors, generalizing the two examples considered in $\S 3.1$. The open Calabi-Yau manifolds underlying the two examples are in fact mirror to each other, as will be readily apparent. In complex dimension 3 these examples are instances of the two types of "trivalent vertices" that typically arise in the discriminant loci of special Lagrangian fibrations on Calabi-Yau 3-folds and appear all over the relevant literature (see e.g. [16]). These examples can also be understood by applying the general machinery developed by Gross and Siebert [18, 19]; nonetheless, we find it interesting to have a fairly explicit and self-contained description of the construction.

Example 3.3.1. Consider $X=\mathbb{C}^{n}$, equipped with the standard Kähler form $\omega$ and the holomorphic volume form $\Omega=\left(\prod x_{i}-\epsilon\right)^{-1} d x_{1} \wedge \cdots \wedge d x_{n}$, which has poles along the hypersurface $D=\left\{\prod x_{i}=\epsilon\right\}$. Then $X \backslash D$ carries a fibration by special Lagrangian tori $T_{r, \lambda}=\left\{\left(x_{1}, \ldots, x_{n}\right) \in \mathbb{C}^{n},\left|\prod x_{i}-\epsilon\right|=\right.$ $\left.r, \mu_{T^{n-1}}\left(x_{1}, \ldots, x_{n}\right)=\lambda\right\}$, where $\mu_{T^{n-1}}: \mathbb{C}^{n} \rightarrow \mathbb{R}^{n-1}$ is the moment map for the action of the group $T^{n-1}=\left\{\operatorname{diag}\left(e^{i \theta_{1}}, \ldots, e^{i \theta_{n}}\right), \sum \theta_{i}=0\right\}$. More 
explicitly,

$T_{r, \lambda}=\left\{\left(x_{1}, \ldots, x_{n}\right) \in \mathbb{C}^{n},\left|\prod_{1}^{n} x_{i}-\epsilon\right|=r, \frac{1}{2}\left(\left|x_{i}\right|^{2}-\left|x_{n}\right|^{2}\right)=\lambda_{i} \forall i=1, \ldots, n-1\right\}$.

The tori $T_{r, \lambda}$ are $T^{n-1}$-invariant, and as in previous examples they are obtained by lifting special Lagrangian fibrations on the reduced spaces. As in Example 3.1.1, these tori are easiest to visualize in terms of the projection $f:\left(x_{1}, \ldots, x_{n}\right) \mapsto \prod x_{i}$, with respect to which they fiber over circles centered at $\epsilon$; see Figure 1 . The main difference is that $f^{-1}(0)$ is now the union of the $n$ coordinate hyperplanes, and $T_{r, \lambda}$ is singular whenever it hits the locus where the $T^{n-1}$-action is not free, namely the points where at least two coordinates vanish. Concretely, $T_{r, \lambda}$ is singular if and only if $r=|\epsilon|$ and $\lambda$ lies in the tropical hyperplane consisting of those $\lambda=\left(\lambda_{1}, \ldots, \lambda_{n-1}\right)$ such that either $\min \left(\lambda_{i}\right)=0$, or $\min \left(\lambda_{i}\right)$ is attained twice. (For $n=3$ this is the union of the three half-lines $0=\lambda_{1} \leq \lambda_{2}, 0=\lambda_{2} \leq \lambda_{1}$, and $\lambda_{1}=\lambda_{2} \leq 0$.)

By the maximum principle, any holomorphic disc in $\left(\mathbb{C}^{n}, T_{r, \lambda}\right)$ which does not intersect $D=f^{-1}(\epsilon)$ must be contained inside a fiber of $f$. The regular fibers of $f$ are diffeomorphic to $\left(\mathbb{C}^{*}\right)^{n-1}$, inside which product tori do not bound any nonconstant holomorphic discs. Hence, $T_{r, \lambda}$ bounds nontrivial Maslov index 0 holomorphic discs if and only if $r=|\epsilon|$. In that case, $T_{|\epsilon|, \lambda}$ intersects one of the components of $f^{-1}(0)$ (i.e. a coordinate hyperplane isomorphic to $\mathbb{C}^{n-1}$ ) in a product torus, which bounds various families of holomorphic discs inside $f^{-1}(0)$.

The wall $r=|\epsilon|$ divides the moduli space of special Lagrangians into two chambers. In the chamber $r>|\epsilon|$, the tori $T_{r, \lambda}$ can be be deformed into product tori by a Hamiltonian isotopy that does not intersect $f^{-1}(0)$ (from the perspective of the projection $f$, the isotopy amounts simply to deforming the circle of radius $r$ centered at $\epsilon$ to a circle of the appropriate size centered at the origin). The product torus $S^{1}\left(r_{1}\right) \times \cdots \times S^{1}\left(r_{n}\right)$ bounds $n$ families of Maslov index 2 discs parallel to the $x_{1}, \ldots, x_{n}$ coordinate axes; denote their classes by $\beta_{1}, \ldots, \beta_{n}$, and by $z_{i}=\exp \left(-\int_{\beta_{i}} \omega\right) \operatorname{hol}_{\nabla}\left(\partial \beta_{i}\right)$ the corresponding holomorphic weights. Thus we expect that $T_{r, \lambda}$ bounds $n$ families of Maslov index 2 holomorphic discs; these are all sections of $f$ over the disc of radius $r$ centered at $\epsilon$, and the discs in the class $\beta_{i}$ intersect the fiber $f^{-1}(0)$ at a point of the coordinate hyperplane $x_{i}=0$. Since the deformation from $T_{r, \lambda}$ to the product torus does not involve any wall-crossing, the count of discs in the class $\beta_{i}$ is 1 , and the superpotential is given by $W=z_{1}+\cdots+z_{n}$.

Next we look at the chamber $r<|\epsilon|$. We first observe that the Chekanovtype torus $T_{r, 0}$ bounds only one family of Maslov index 2 holomorphic discs. Indeed, since Maslov index 2 discs have intersection number 1 with $D=f^{-1}(\epsilon)$, they must be sections of $f$ over the disc of radius $r$ centered at $\epsilon$, and hence they do not intersect any of the coordinate hyperplanes. However, on $T_{r, 0}$ we have $\left|x_{1}\right|=\cdots=\left|x_{n}\right|$, so the maximum principle applied to $x_{i} / x_{n}$ implies that the various coordinates $x_{i}$ are proportional to each other, i.e. all such holomorphic discs must be contained in lines passing through 
the origin. One easily checks that this gives a single family of holomorphic discs; we denote by $\beta_{0}$ the corresponding homotopy class and by $u=z_{\beta_{0}}$ the corresponding weight. Finally, since no exceptional discs arise in the deformation of $T_{r, 0}$ to $T_{r, \lambda}$, we deduce that $T_{r, \lambda}$ also bounds a single family of holomorphic discs in the class $\beta_{0}$, and that the superpotential in the chamber $r<|\epsilon|$ is given by $W=u$.

When we increase the value of $r$ past $r=|\epsilon|$, with all $\lambda_{i}>0$, the torus $T_{r, \lambda}$ crosses the coordinate hyperplane $x_{n}=0$, and the family of holomorphic discs in the class $\beta_{0}$ naturally deforms into the family of discs in the class $\beta_{n}$ mentioned above. However, the naive gluing $u=z_{n}$ must be corrected by wall-crossing contributions. For $r=|\epsilon|, T_{r, \lambda}$ intersects the hyperplane $x_{n}=0$ in a product torus. This torus bounds $n-1$ families of discs parallel to the coordinate axes inside $\left\{x_{n}=0\right\}$, whose classes we denote by $\alpha_{1}, \ldots, \alpha_{n-1}$; we denote by $w_{1}, \ldots, w_{n-1}$ the corresponding holomorphic weights, which satisfy $\left|w_{i}\right|=e^{-\lambda_{i}}$. It is easy to check that, on the $r>|\epsilon|$ side, we have $\alpha_{i}=\beta_{i}-\beta_{n}$, and hence $w_{i}=z_{i} / z_{n}$; general features of wall-crossing imply that $w_{i}$ should not be affected by instanton corrections. Continuity of the superpotential across the wall implies that the relation between $u$ and $z_{n}$ should be modified to $u=z_{1}+\cdots+z_{n}=z_{n}\left(w_{1}+\cdots+w_{n-1}+1\right)$. Thus, only the families of Maslov index 0 discs in the classes $\alpha_{1}, \ldots, \alpha_{n-1}$ contribute to the instanton corrections, even though the product torus in $\left\{x_{n}=0\right\}$ also bounds higher-dimensional families of holomorphic discs, whose classes are positive linear combinations of the $\alpha_{i}$.

Similarly, when we increase the value of $r$ past $r=|\epsilon|$, with some $\lambda_{k}=$ $\min \left\{\lambda_{i}\right\}<0$, the torus $T_{r, \lambda}$ crosses the coordinate hyperplane $x_{k}=0$, and the family of discs in the class $\beta_{0}$ deforms to the family of discs in the class $\beta_{k}$. However, for $r=|\epsilon|, T_{r, \lambda}$ intersects the hyperplane $x_{k}=0$ in a product torus, which bounds $n-1$ families of discs parallel to the coordinate axes, representing the classes $\alpha_{i}-\alpha_{k}=\beta_{i}-\beta_{k}(i \neq k, n)$, with weight $w_{i} w_{k}^{-1}=z_{i} / z_{k}$, and $-\alpha_{k}=\beta_{n}-\beta_{k}$, with weight $w_{k}^{-1}=z_{n} / z_{k}$. The instanton-corrected gluing is now $u=z_{k}\left(z_{1} / z_{k}+\cdots+z_{n} / z_{k}+1\right)=z_{1}+\cdots+z_{n}$.

Piecing things together as in Example 3.1.1, we obtain a description of the corrected and completed SYZ mirror in terms of the coordinates $u$, $v=z_{n}^{-1}, w_{1}, \ldots, w_{n-1}$ :

Proposition 3.3. The mirror of $X=\mathbb{C}^{n}$ relatively to the divisor $D=$ $\left\{\prod x_{i}=\epsilon\right\}$ is

$$
\begin{aligned}
& X^{\vee}=\left\{\left(u, v, w_{1}, \ldots, w_{n-1}\right) \in \mathbb{C}^{2} \times\left(\mathbb{C}^{*}\right)^{n-1}, u v=1+w_{1}+\cdots+w_{n-1}\right\} \\
& \quad W=u
\end{aligned}
$$

A final remark: one way to check that the variables $w_{i}$ are indeed not affected by the wall-crossing is to compactify $\mathbb{C}^{n}$ to $\left(\mathbb{C P}^{1}\right)^{n}$, equipped now with the standard product Kähler form. Inside $\left(\mathbb{C P}^{1}\right)^{n}$ the tori $T_{r, \lambda}$ also bound families of Maslov index 2 discs that pass through the divisors at infinity. These discs are sections of $f$ over the complement of the disc of 
radius $r$ centered at $\epsilon$, and can be described explicitly in coordinates after deforming $T_{r, \lambda}$ to either a product torus (for $r>|\epsilon|$ ) or a Chekanov torus $T_{r, 0}$ (for $r<|\epsilon|$ ). In the latter case, we notice that the discs intersect the divisor at infinity once and $f^{-1}(0)$ once, so that in affine coordinates exactly one component of the map has a zero and exactly one has a pole. Each of the $n^{2}$ possibilities gives one family of holomorphic discs; the calculations are a straightforward adaptation of the case of $\mathbb{C P}^{1} \times \mathbb{C P}^{1}$ treated in Section 5.4 of [4]. The continuity of $W$ leads to an identity between the contributions to the superpotential coming from discs that intersect the compactification divisor " $x_{k}=\infty$ " (a single family of discs for $r>|\epsilon|$, vs. $n$ families for $r<|\epsilon|)$ : namely, denoting by $\Lambda$ the area of $\mathbb{C P}^{1}$, we must have

$$
\frac{e^{-\Lambda}}{z_{k}}=\frac{e^{-\Lambda}}{u w_{k}}\left(w_{1}+\cdots+w_{n-1}+1\right) .
$$

This is consistent with the formulas given above for the gluing between the two chambers.

EXAMPLE 3.3.2. This example is treated carefully in [2], where it is used as a standard building block to construct mirrors of hypersurfaces in toric varieties. Here we only give an outline, for completeness and for symmetry with the previous example.

Consider $\mathbb{C}^{n}$ equipped with the standard holomorphic volume form $\prod d \log x_{i}$, and blow up the codimension 2 linear subspace $Y \times 0=\left\{x_{1}+\cdots+\right.$ $\left.x_{n-1}=1, x_{n}=0\right\}$. This yields a complex manifold $X$ equipped with the holomorphic volume form $\Omega=\pi^{*}\left(\prod d \log x_{i}\right)$, with poles along the proper transform $D$ of the coordinate hyperplanes. The $S^{1}$-action rotating the last coordinate $x_{n}$ lifts to $X$; consider an $S^{1}$-invariant Kähler form $\omega$ for which the area of the $\mathbb{C P}^{1}$ fibers of the exceptional divisor is $\epsilon(\epsilon \ll 1)$, and which agrees with the standard Kähler form of $\mathbb{C}^{n}$ away from a neighborhood of the exceptional divisor. Denote by $\mu_{S^{1}}: X \rightarrow \mathbb{R}$ the moment map of the $S^{1}$-action, normalized to equal 0 on the proper transform of the coordinate hyperplane $x_{n}=0$, and $\epsilon$ at the stratum of fixed points given by the section "at infinity" of the exceptional divisor.

The reduced spaces $X_{\lambda}=\left\{\mu_{S^{1}}=\lambda\right\} / S^{1}(\lambda \geq 0)$ are all smooth and diffeomorphic to $\mathbb{C}^{n-1}$. They carry natural holomorphic volume forms, which are the pullbacks of $d \log x_{1} \wedge \cdots \wedge d \log x_{n-1}$, and Kähler forms $\omega_{\lambda}$. While $\omega_{\lambda}$ agrees with the standard Kähler form for $\lambda \gg \epsilon$, for $\lambda<\epsilon$ the form $\omega_{\lambda}$ is not toric; rather, it can be described as the result of collapsing a tubular neighborhood of size $\epsilon-\lambda$ of the hypersurface $Y=\left\{x_{1}+\cdots+x_{n-1}=1\right\}$ inside the standard $\mathbb{C}^{n-1}$. Thus, it is not entirely clear that $X_{\lambda}$ carries a special Lagrangian torus fibration (though it does seem likely).

Nonetheless, using Moser's theorem to see that $\omega_{\lambda}$ is symplectomorphic to the standard form on $\mathbb{C}^{n-1}$, we can find a Lagrangian torus fibration on the complement of the coordinate hyperplanes in $\left(X_{\lambda}, \omega_{\lambda}\right)$. Taking the preimages of these Lagrangians in $\left\{\mu_{S^{1}}=\lambda\right\}$, we obtain a Lagrangian fibration on 
$X \backslash D$, whose fibers are $S^{1}$-invariant Lagrangian tori $L_{r, \lambda}$; for $\lambda \gg \epsilon$ these tori are of the form

$$
\left\{\left|\pi^{*}\left(x_{i}\right)\right|=r_{i} \forall 1 \leq i \leq n-1, \quad \mu_{S^{1}}=\lambda\right\} .
$$

The singularities of this fibration correspond to the fixed points of the $S^{1}$-action inside $X \backslash D$, namely the "section at infinity" of the exceptional divisor, defined by the equations $\left\{\mu_{S^{1}}=\epsilon, \pi^{*} x_{1}+\cdots+\pi^{*} x_{n-1}=1\right\}$. In the base of the fibration, the discriminant locus is therefore of real codimension 1 , namely the amoeba of the hypersurface $Y$, sitting inside the affine hyperplane $\lambda=\epsilon$ (see Figure 5 left). Moreover, $L_{r, \lambda}$ bounds nonconstant discs of Maslov index 0 if and only if it contains points where $\pi^{*} x_{1}+\cdots+\pi^{*} x_{n-1}=1$. In that case, the Maslov index 0 discs are contained in the total transforms of lines parallel to the $x_{n}$-axis passing through a point of $Y \times 0$. Thus, there are $n+1$ regions in which the tori $L_{r, \lambda}$ are weakly unobstructed, corresponding to the connected components of the complement of the amoeba of $Y$.

To analyze holomorphic discs in $\left(X, L_{r, \lambda}\right)$ and their contributions to the superpotential, we consider tori which lie far away from the exceptional divisor and from the walls, i.e. for $r=\left(r_{1}, \ldots, r_{n-1}\right)$ sufficiently far from the amoeba of $Y$; then $L_{r, \lambda}$ projects to a product torus in $\mathbb{C}^{n}$. When all $r_{i} \ll 1$ for all $i$, the maximum principle implies that holomorphic discs bounded by $L_{r, \lambda}$ cannot hit the exceptional divisor; hence $L_{r, \lambda}$ bounds $n$ families of Maslov index 2 holomorphic discs, parallel to the coordinate axes. Denote by $\beta_{1}, \ldots, \beta_{n-1}, \delta$ the classes of these discs, and by $u_{1}, \ldots, u_{n-1}, z$ the corresponding weights (i.e., the complexifications of the affine coordinates pictured in the lower-left chamber of Figure 5 right).

Next consider the case where $r_{k} \gg 1$ and $r_{k} \gg r_{i} \forall i \neq k$. Then we claim that $L_{r, \lambda}$ now bounds $n+1$ families of Maslov index 2 holomorphic discs. Namely, since a Maslov index 2 disc intersects $D$ exactly once, and the projections to the coordinates $\left(x_{1}, \ldots, x_{n}\right)$ are holomorphic, at most one of $\pi^{*}\left(x_{1}\right), \ldots, \pi^{*}\left(x_{n-1}\right)$ can be non-constant over such a disc. Arguing as in the 2-dimensional case (Example 3.1.2), we deduce that $L_{r, \lambda}$ bounds $n$ families of discs parallel to the coordinate axes, and one additional family, namely the proper transforms of Maslov index 4 discs in $\mathbb{C}^{n}$ which are parallel to the $\left(x_{k}, x_{n}\right)$-plane and hit the hyperplane $x_{n}=0$ at a point of $Y$. Denote by $u_{1,(k)}, \ldots, u_{n-1,(k)}, z_{(k)}$ the weights associated to the first $n$ families of
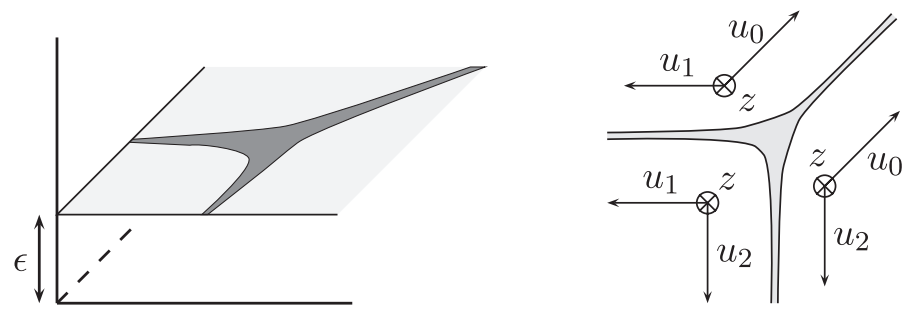

FiguRE 5. $\mathbb{C}^{3}$ blown up along $\left\{x_{1}+x_{2}=1, x_{3}=0\right\}$ 
discs: then the contribution of the additional family to the superpotential is $e^{\epsilon} z_{(k)} u_{k,(k)}$.

Matching the contributions of the families of discs that intersect each component of $D$, we conclude that the instanton-corrected gluings are given by $z=z_{(k)}, u_{i}=u_{i,(k)}$ for $i \neq k$, and $u_{k}=u_{k,(k)}\left(1+e^{\epsilon} z\right)$. Let

$$
u_{0,(k)}=\left(\prod_{i=1}^{n-1} u_{i,(k)}\right)^{-1}=\left(\prod_{i=1}^{n-1} u_{i}\right)^{-1}\left(1+e^{\epsilon} z\right) .
$$

Then the coordinate $u_{0,(k)}$ is independent of $k$, and we can denote it simply by $u_{0}$. The coordinates $\left(u_{0}, \ldots, u_{n-1}, z\right)$ can now be used to give a global description of the mirror (since forgetting one of the $u_{i}$ gives a set of coordinates for each chamber, as depicted in Figure 5 right). Namely, after completion we arrive at:

Proposition 3.4 (Abouzaid,-, Katzarkov [2]). The SYZ mirror of the blowup of $\mathbb{C}^{n}$ along $\left\{x_{1}+\cdots+x_{n-1}=1, x_{n}=0\right\}$ with anticanonical divisor the proper transform of the toric divisor is

$$
\begin{aligned}
X^{\vee} & =\left\{\left(u_{0}, \ldots, u_{n-1}, z\right) \in \mathbb{C}^{n} \times \mathbb{C}^{*}, u_{0} \ldots u_{n-1}=1+e^{\epsilon} z\right\}, \\
W & =u_{1}+\cdots+u_{n-1}+z .
\end{aligned}
$$

If instead we consider the blowup of $\left(\mathbb{C}^{*}\right)^{n-1} \times \mathbb{C}$ along the generalized pair of pants $\left\{x_{1}+\cdots+x_{n-1}=1, x_{n}=0\right\}$, i.e. we remove all the components of $D$ except the proper transform of the $x_{n}=0$ coordinate hyperplane, then $X^{\vee}$ remains the same but the superpotential becomes simply $W=z$ (since all the other terms in the above formula correspond to discs that intersect the coordinate hyperplanes that we are now removing).

In $[\mathbf{2}]$, these local models are patched together in order to build mirrors of more complicated blowups. The motivation for such a construction comes from the observation that, if $Y$ is a hypersurface in $X$, then the derived category of $Y$ embeds into that of the blowup of $X \times \mathbb{C}$ along $Y \times 0$ (this follows from a more general theorem of Bondal and Orlov, see e.g. [7]); and, if $Y$ deforms in a pencil, then the Fukaya categories of these two manifolds are also closely related (using Seidel's work; the key point is that Lefschetz thimbles for a pencil in $X$ can be lifted to Lagrangian spheres in the blowup of $X \times \mathbb{C}$ along $Y$ ). Thus, a mirror for the blowup of $X \times \mathbb{C}$ along $Y$ is almost as good as a mirror for $Y$. We illustrate this by considering one half of the homological mirror symmetry conjecture in a very simple example.

Consider the case $n=3$ of Proposition 3.4 and its variants where we remove various divisors from $D$. Consider the blowup of $\left(\mathbb{C}^{*}\right)^{2} \times \mathbb{C}$ along $\left\{x_{1}+\right.$ $\left.x_{2}=1, x_{1}, x_{2} \neq 0\right\}$ (a pair of pants, i.e. $\mathbb{P}^{1}$ minus three points): then $X^{\vee}$ is as in Proposition 3.4, i.e. (solving for $z$ as a function of $u_{0}, u_{1}, u_{2}$ ) the complement of the hypersurface $u_{0} u_{1} u_{2}=1$ inside $\mathbb{C}^{3}$, and the superpotential is $W=z=e^{-\epsilon}\left(u_{0} u_{1} u_{2}-1\right)$, whose critical locus consists of the union of the three coordinate axes. Up to an irrelevant scaling of the superpotential, this 
Landau-Ginzburg model is indeed known to be a mirror to the pair of pants (cf. work of Abouzaid and Seidel; see also [38]). If instead we consider the blowup of $\mathbb{C}^{*} \times \mathbb{C}^{2}$ along $\left\{x_{1}+x_{2}=1, x_{1} \neq 0\right\}\left(\cong \mathbb{C}^{*}\right)$, then the superpotential becomes $W=u_{2}+z=\left(e^{-\epsilon} u_{0} u_{1}+1\right) u_{2}-e^{-\epsilon}$; hence $W$ has a Morse-Bott singularity along $M=\left\{u_{0} u_{1}=-e^{\epsilon}, u_{2}=0\right\} \simeq \mathbb{C}^{*}$, which is mirror to $\mathbb{C}^{*}$. Finally, if we compactify our example to consider the blowup of $\mathbb{C P}^{2} \times \mathbb{C}$ along a projective line (given by $x_{1}+x_{2}=1$ in affine coordinates), then the mirror remains the same manifold, but the superpotential acquires an extra term counting discs that pass through the divisor at infinity, and becomes

$$
W=e^{-\Lambda} u_{0}+u_{1}+u_{2}+z=e^{-\Lambda} u_{0}+u_{1}+u_{2}+e^{-\epsilon} u_{0} u_{1} u_{2}-e^{-\epsilon}
$$

where $\Lambda$ is the area of a line in $\mathbb{C P}^{2}$. This superpotential has two isolated non-degenerate critical points at $e^{-\Lambda} u_{0}=u_{1}=u_{2}=e^{ \pm i \pi / 2} e^{(\epsilon-\Lambda) / 2}$, which is reminiscent of the usual mirror of a $\mathbb{C P}^{1}$ with symplectic area $\Lambda-\epsilon$ (to which our mirror can be related by Knörrer periodicity).

\section{Floer-theoretic considerations}

4.1. Deformations and local systems. There are at least three possible ways of deforming the Floer theory of a given Lagrangian submanifold $L$ (for simplicity we assume $L$ to be weakly unobstructed):

(1) formally deforming the Floer theory of $L$ by an element $b \in C F^{1}(L, L)$;

(2) equipping $L$ with a non-unitary local system;

(3) deforming $L$ by a (non-Hamiltonian) Lagrangian isotopy and equipping it with a unitary local system.

Our goal in this paragraph is to explain informally how these three flavors of deformation are related. In particular, the careful reader will notice that Fukaya-Oh-Ohta-Ono define the superpotential as a function on the moduli space of weak bounding cochains for a given Lagrangian $[\mathbf{1 4}, \mathbf{1 5}]$, following the first approach, whereas in this paper and in [4] we view it as a function on a moduli space of Lagrangians equipped with unitary local systems, following the last approach.

Recall that there are several models for the Floer complex $C F^{*}(L, L)$. We mostly consider the version in [14], where the Floer complex is generated by singular chains on $L$, representing incidence conditions at marked points on the boundary of holomorphic discs. The $k$-fold product $\mathfrak{m}_{k}$ is defined by

$$
\begin{aligned}
& \mathfrak{m}_{k}\left(C_{1}, \ldots, C_{k}\right) \\
& \quad=\sum_{\beta \in \pi_{2}(X, L)} z_{\beta}(L)\left(e v_{0}\right)_{*}\left(\left[\overline{\mathcal{M}}_{k+1}(L, \beta)\right]^{v i r} \cap e v_{1}^{*} C_{1} \cap \cdots \cap e v_{k}^{*} C_{k}\right),
\end{aligned}
$$

where $\left[\overline{\mathcal{M}}_{k+1}(L, \beta)\right]^{\text {vir }}$ is the (virtual) fundamental chain of the moduli space of holomorphic discs in $(X, L)$ with $k+1$ boundary marked points representing the class $\beta, e v_{0}, \ldots, e v_{k}$ are the evaluation maps at the marked points, 
and $z_{\beta}$ is a weight factor as in $(2.2)$; when $k=1$ the term with $\beta=0$ is replaced by a classical boundary term.

Here it is useful to also keep in mind a variant where the Floer complex consists of differential forms or currents on $L$. The product $\mathfrak{m}_{k}$ is defined as in (4.1), which now involves pulling back the given forms/currents to the moduli space of discs via the evaluation maps $e v_{1}, \ldots, e v_{k}$ and pushing forward their product by integration along the fibers of $e v_{0}$. This setup allows us to "smudge" incidence conditions by replacing the integration current on a submanifold $C_{i}$ by a smooth differential form supported in a tubular neighborhood.

Given $b \in C F^{1}(L, L)$, Fukaya-Oh-Ohta-Ono [14] deform the $A_{\infty}$-algebra structure on the Floer complex by setting

$$
\mathfrak{m}_{k}^{b}\left(C_{1}, \ldots, C_{k}\right)=\sum_{l=l_{0}+\cdots+l_{k} \geq 0} \mathfrak{m}_{k+l}(\underbrace{b, \ldots, b}_{l_{0}}, C_{1}, \underbrace{b, \ldots, b}_{l_{1}}, \ldots, C_{k}, \underbrace{b, \ldots, b}_{l_{k}}) .
$$

We will actually restrict our attention to the case where $b$ is a cycle, representing a class $[b] \in H^{1}(L)$ (or, dually, in $H_{n-1}(L)$ ).

Working over the Novikov ring, the sum (4.2) is guaranteed to be welldefined when $b$ has coefficients in the maximal ideal

$$
\Lambda_{+}=\left\{\sum_{i} a_{i} T^{\lambda_{i}} \in \Lambda_{0} \mid \lambda_{i}>0\right\}
$$

of $\Lambda_{0}=\left\{\sum a_{i} T^{\lambda_{i}} \mid a_{i} \in \mathbb{C}, \lambda_{i} \in \mathbb{R}_{\geq 0}, \lambda_{i} \rightarrow+\infty\right\}$. However, it has been observed by Cho [10] (see also [15]) that, in the toric case, the sum (4.2) is convergent even when $b$ is a general element of $H^{1}\left(L, \Lambda_{0}\right)$. Similarly, in favorable cases (at least for toric Fanos) we can also hope to make sense of (4.2) when working over $\mathbb{C}$ (in the "convergent power series" setting); however in general this poses convergence problems.

The second type of deformation we consider equips $L$ with a local system (a flat connection), characterized by its holonomy hol $\nabla$, which is a homomorphism from $\pi_{1}(L)$ to $\Lambda_{0}^{*}$ (the multiplicative group formed by elements of the Novikov ring with nonzero coefficient of $T^{0}$ ) or $\mathbb{C}^{*}$. The local system modifies the weight $z_{\beta}$ for the contribution to $\mathfrak{m}_{k}$ of discs in the class $\beta$ by a factor of $\operatorname{hol}_{\nabla}(\partial \beta)$.

Lemma 4.1. For any cycle $b$ such that convergence holds, the deformation of the $A_{\infty}$-algebra $C F^{*}(L, L)$ given by (4.2) is equivalent to equipping $L$ with a local system with holonomy $\exp (b)$, i.e. such that $\operatorname{hol}_{\nabla}(\gamma)=$ $\exp ([b] \cdot[\gamma])$ for all $\gamma \in \pi_{1}(L)$. 
SkETCH OF PROOF. The statement reduces to a calculation showing that, given a holomorphic disc $u \in \mathcal{M}_{k+1}(L, \beta)$ (or more generally an element of the compactified moduli space), the contribution of "refined" versions of $u$ (with extra marked points mapped to $b$ ) to $\mathfrak{m}_{k}^{b}$ is $\exp ([b] \cdot[\partial \beta]$ ) times the contribution of $u$ to $\mathfrak{m}_{k}$.

This is easiest to see when we represent the class [b] by a smooth closed 1-form on $L$. For fixed $\underline{l}=\left(l_{0}, \ldots, l_{k}\right)$, consider the forgetful map $\pi_{\underline{l}}: \mathcal{M}_{k+l+1}(L, \beta) \rightarrow \mathcal{M}_{k+1}(L, \beta)$ which deletes the marked points corresponding to the $b$ 's in (4.2), and its extension $\bar{\pi}_{\underline{l}}$ to the compactified moduli spaces. The fiber of $\pi_{l}$ above $u \in \mathcal{M}_{k+1}(L, \beta)$ is a product of open simplices of dimensions $l_{0}, \ldots, \bar{l}_{k}$, parametrizing the positions of the $l_{0}+\cdots+l_{k}$ new marked points along the intervals separated by the $k+1$ marked points of $u$ on the boundary of the disc; we denote by $\Delta_{\underline{l}}$ the corresponding subset of $\left(\partial D^{2}\right)^{l}$. The formula for $\mathfrak{m}_{k+l}\left(b^{\otimes l_{0}}, C_{1}, b^{\otimes l_{1}}, \ldots, C_{k}, b^{\otimes l_{k}}\right)$ involves an integral over $\overline{\mathcal{M}}_{k+l+1}(L, \beta)$, but this integral can be pushed forward to $\overline{\mathcal{M}}_{k+1}(L, \beta)$ by integrating over the fibers of $\bar{\pi}_{l}$; the resulting integral differs from that for $\mathfrak{m}_{k}\left(C_{1}, \ldots, C_{k}\right)$ by an extra factor $\int_{\bar{\pi}_{l}^{-1}(u)} \prod e v_{i}^{*} b=\int_{\bar{\Delta}_{\underline{l}}} \prod\left(u_{\mid \partial D^{2}} \circ p r_{i}\right)^{*} b$ in the integrand.

Note that this calculation assumes that the virtual fundamental chains have been constructed consistently, so that $\left[\overline{\mathcal{M}}_{k+l+1}(L, \beta)\right]^{v i r}=\bar{\pi}_{l}^{*}\left(\left[\overline{\mathcal{M}}_{k+1}\right.\right.$ $\left.(L, \beta)]^{v i r}\right)$ as expected. Achieving this property is in general a non-trivial problem.

Next we sum over $\underline{l}$ : the subsets $\bar{\Delta}_{\underline{l}}$ of $\left(\partial D^{2}\right)^{l}$ have disjoint interiors, and their union $\bar{\Delta}$ is the set of all $l$-tuples of points which lie in counterclockwise order on the interval obtained by removing the outgoing marked point of $u$ from $\partial D^{2}$. By symmetry, the integral of $\prod\left(u_{\mid \partial D^{2}} \circ p r_{i}\right)^{*} b$ over $\bar{\Delta}$ is $1 / l$ ! times the integral over $\left(\partial D^{2}\right)^{l}$. Thus

$$
\begin{aligned}
\sum_{\underline{l}} \int_{\bar{\Delta}_{\underline{\underline{l}}}} \prod_{i=1}^{l}\left(u \circ p r_{i}\right)^{*} b & =\frac{1}{l !} \int_{\left(\partial D^{2}\right)^{l}} \prod_{i=1}^{l}\left(u \circ p r_{i}\right)^{*} b=\frac{1}{l !}\left(\int_{\partial D^{2}} u^{*} b\right)^{l} \\
& =\frac{([b] \cdot[\partial \beta])^{l}}{l !} .
\end{aligned}
$$

The statement then follows by summing over $l$.

One can also try to prove Lemma 4.1 working entirely with chains on $L$ instead of differential forms, but it is technically harder. If we take $b$ to be a codimension 1 cycle in $L$ and attempt to reproduce the above argument, the incidence constraints at the additional marked points (all mapping to $b$ ) are not transverse to each other. In fact, $\mathfrak{m}_{k}^{b}$ will include contributions from stable maps with constant disc bubbles mapping to $b$. The difficulty is then to understand the combinatorial rule for counting such contributions, or more precisely, why a constant bubble with $j$ marked points on it, all mapped to a same point of $b$, should contribute a combinatorial factor of $1 / j !$. 
The equivalence between the two types of deformations also holds if we consider not just $L$ itself, but the whole Fukaya category. Given a collection of Lagrangian submanifolds $L_{0}, \ldots, L_{k}$ with $L_{i_{0}}=L$ for some $i_{0}$, the Floer theoretic product $\mathfrak{m}_{k}: C F^{*}\left(L_{0}, L_{1}\right) \otimes \cdots \otimes C F^{*}\left(L_{k-1}, L_{k}\right) \rightarrow C F^{*}\left(L_{0}, L_{k}\right)$ can again be deformed by a cycle $b \in C F^{1}(L, L)$. Where the usual product $\mathfrak{m}_{k}$ is a sum over holomorphic discs with $k+1$ marked points, the deformed product $\mathfrak{m}_{k}^{b}$ counts discs with an arbitrary number of additional marked points, all lying on the interval of $\partial D^{2}$ which gets mapped to $L$, and with inputs $b$ inserted accordingly into the Floer product as in (4.2). By the same argument as above, if we represent $b$ by a closed 1 -form on $L$, and consider discs with fixed corners and in a fixed homotopy class $\beta$, the deformation amounts to the insertion of an extra factor $\exp \left(\int_{\partial \beta \cap L} b\right)$. Meanwhile, equipping $L$ with a flat connection $\nabla$ affects the count of discs in the class $\beta$ by a factor $\operatorname{hol}_{\nabla}(\partial \beta \cap L)$. Thus, if we ensure that the two match, e.g. by choosing $\nabla=d+b$, the two deformations are again equivalent.

Next, we turn to the relation between non-unitary local systems and non Hamiltonian deformations. Consider a deformation of $L$ to a nearby Lagrangian submanifold $L_{1}$; identifying a tubular neighborhood of $L$ with a neighborhood of the zero section in $T^{*} L$, we can think of $L_{1}$ as the graph of a $C^{1}$-small closed form $\varphi \in \Omega^{1}(L, \mathbb{R})$. Assume that $L$ can be isotoped to $L_{1}$ (e.g. through $\left.L_{t}=\operatorname{graph}(t \varphi)\right)$ in such a way that there is a one-to-one correspondence between the holomorphic discs bounded by $L$ and $L_{1}$, namely given a class $\beta \in \pi_{2}(X, L)$ and the corresponding class $\beta_{1} \in \pi_{2}\left(X, L_{1}\right)$, we have $\mathcal{M}_{k+1}(L, \beta) \simeq \mathcal{M}_{k+1}\left(L_{1}, \beta_{1}\right)$. Observing that $\int_{\beta_{1}} \omega=\int_{\beta} \omega+\int_{\partial \beta} \varphi$, deforming $L$ to $L_{1}$ affects the contribution of these discs by a factor of $\exp (-[\varphi] \cdot[\partial \beta])$. Thus, deforming $L$ to $L_{1}$ is equivalent to equipping $L$ with a local system with holonomy $\exp (-[\varphi])$; for example we can set $\nabla=d-\varphi$. (This is when working over complex numbers; over the Novikov ring we would similarly want to equip $L$ with a local system with holonomy $T^{[\varphi]}$.) However, this only works as long as there is a good correspondence between moduli spaces of holomorphic discs; while the assumption we made can be relaxed to some extent, we cannot expect things to work so simply when the deformation from $L$ to $L_{1}$ involves wall-crossing.

Similarly, given another Lagrangian submanifold $L^{\prime}$, if the isotopy from $L$ to $L_{1}=\operatorname{graph}(\varphi)$ can be carried out in a manner that remains transverse to $L^{\prime}$ at all times then we can hope to define a map from $C F^{*}\left((L, \nabla), L^{\prime}\right)$ (with $\operatorname{hol}(\nabla)=\exp (-[\varphi]))$ to $C F^{*}\left(L_{1}, L^{\prime}\right)$ in a manner compatible with all Floer-theoretic products as long as we can find a one-to-one correspondence between the relevant holomorphic discs. One could also try to proceed in a slightly greater degree of generality by attempting to construct continuation maps between the Floer complexes (as in the usual proof of Hamiltonian isotopy invariance of Floer homology). However, one should keep in mind that this is doomed to fail in general. For instance, consider $X=S^{2}$, let $L_{1}$ be the equator, and $L$ a circle parallel to the equator but disjoint from it. Denote by $A$ the annulus bounded by $L$ and $L_{1}$, and equip $L$ with a non-unitary local 
system $\nabla$ with holonomy $\exp \left(\int_{A} \omega\right)$. One easily checks that the Lagrangians $L_{1}$ and $(L, \nabla)$ have well-defined and non-vanishing Floer homology, and the $A_{\infty}$-algebras $C F^{*}\left(L_{1}, L_{1}\right)$ and $C F^{*}((L, \nabla),(L, \nabla))$ are isomorphic (by the argument above). However, $C F^{*}\left((L, \nabla), L_{1}\right)=0$ since $L$ and $L_{1}$ are disjoint, so $(L, \nabla)$ and $L_{1}$ cannot be isomorphic. (See also the discussion in $\S 4.2$ ).

Remark. Specializing (4.2) to $k=0$, the identity $\mathfrak{m}_{0}^{b}=\mathfrak{m}_{0}+\mathfrak{m}_{1}(b)+$ $\mathfrak{m}_{2}(b, b)+\ldots$ offers a simple perspective into the idea that the derivatives of the superpotential $W$ at a point $\mathcal{L}=(L, \nabla)$ encode information about the (symmetrized) Floer products $\mathfrak{m}_{k}$ on $C F^{*}(\mathcal{L}, \mathcal{L})$, as first shown by Cho in [9]. In particular, one can re-derive from this identity the fact that, if $\mathcal{L}$ is not a critical point of the superpotential, then the fundamental class of $L$ is a Floer coboundary and $H F^{*}(\mathcal{L}, \mathcal{L})$ vanishes. (For a direct proof, see $[\mathbf{1 1}, \mathbf{9}]$, see also $\S 6$ of $[\mathbf{4}]$.)

4.2. Failure of invariance and divergence issues. In this section, we look more carefully into a subtle issue with instanton corrections and the interpretation of the mirror as a moduli space of Lagrangian submanifolds up to Floer-theoretic equivalence. We return to Example 3.1.1, i.e. $\mathbb{C}^{2}$ equipped with the standard Kähler form and the holomorphic volume form $\Omega=d x \wedge d y /(x y-\epsilon)$, and use the same notations as above. Consider two special Lagrangian fibers on opposite sides of the wall, $T_{1}=T_{r_{1}, 0}$ and $T_{2}=T_{r_{2}, 0}$, where $r_{1}<|\epsilon|<r_{2}$ are chosen in a way such that the points of $M$ corresponding to $T_{1}$ and $T_{2}$ (equipped with the trivial local systems) are identified under the instanton-corrected gluing $u=z_{1}+z_{2}$. Namely, the torus $T_{1}$ corresponds to a point with coordinates $w=1, u=\exp \left(-A_{1}\right) \in \mathbb{R}_{+}$, where $A_{1}$ is the symplectic area of a Maslov index 2 disc in $\left(\mathbb{C}^{2}, T_{1}\right)$, e.g. either of the two portions of the line $x=y$ where $|x y-\epsilon|<r_{1}$; meanwhile, $T_{2}$ corresponds to $w=1, z_{1}=z_{2}=\exp \left(-A_{2}\right) \in \mathbb{R}_{+}$, where $A_{2}$ is the symplectic area of a Maslov index 2 disc in $\left(\mathbb{C}^{2}, T_{2}\right)$, or equivalently half of the area of the portion of the line $x=y$ where $|x y-\epsilon|<r_{2}$. The area $A_{i}$ can be expressed by an explicit formula in terms of $r_{i}$ and $\epsilon$; the actual relation is irrelevant, all that matters to us is that $A_{i}$ is a monotonically increasing function of $r_{i}$. Now we choose $r_{1}$ and $r_{2}$ such that $\exp \left(-A_{1}\right)=2 \exp \left(-A_{2}\right)$ and $r_{1}<|\epsilon|<r_{2}$.

We will consider the tori $T_{1}$ and $T_{2}$ inside $X^{0}=X \backslash D=\mathbb{C}^{2} \backslash\{x y=\epsilon\}$, where they do not bound any nonconstant holomorphic discs. (Another option would be to instead compactify $\mathbb{C}^{2}$ to $\mathbb{C P}^{2}$, and choose the parameters of the construction so that $\exp \left(-A_{1}\right)=2 \exp \left(-A_{2}\right)=\exp \left(-\frac{1}{3} \int_{\mathbb{C P}^{1}} \omega\right)$; then $T_{1}$ and $T_{2}$ would be weakly unobstructed and would still have non-vanishing convergent power series Floer homology. The discussion below would carry over with minor modifications.)

Working in $X^{0}$, the convergent power series Floer homologies $H F^{*}$ $\left(T_{1}, T_{1}\right)$ and $H F^{*}\left(T_{2}, T_{2}\right)$ are isomorphic to each other (and to the cohomology of $T^{2}$ ). In fact the same property would hold for any other $T_{r, \lambda}$ due to the absence of holomorphic discs in $X^{0}$, but in the case of $T_{1}$ and $T_{2}$ 

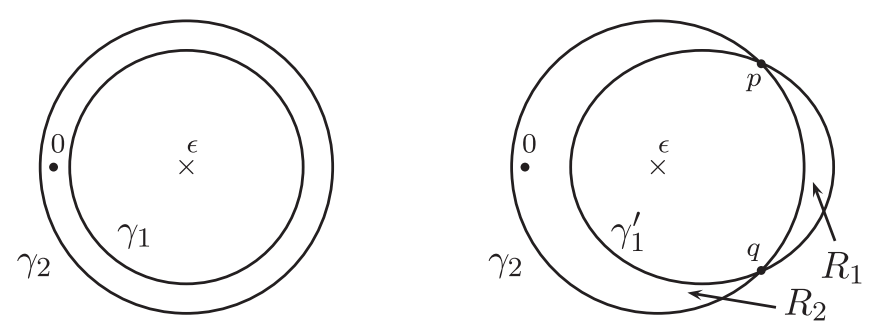

Figure 6 . Creating intersections between $T_{1}$ and $T_{2}$

we expect to have a distinguished isomorphism between the Floer homology groups, considering that $T_{1}$ and $T_{2}$ are in the same instanton-corrected equivalence class and meant to be "isomorphic". However, $T_{1}$ and $T_{2}$ are disjoint, so $C F^{*}\left(T_{1}, T_{2}\right)$ is zero, which does not allow for the existence of the expected isomorphism. (Note that the issue would not arise when working over the Novikov ring: we would then have needed to choose the areas $A_{1}$ and $A_{2}$ above so that $T^{-A_{1}}=2 T^{-A_{2}}$, which never holds. In that case, one should instead take $A_{1}=A_{2}$ and equip $T_{1}$ with a nontrivial local system; but then $T_{1}$ and $T_{2}$ cannot be made disjoint by Hamiltonian isotopies.)

One way to realize the isomorphism between $T_{1}$ and $T_{2}$ is to deform one of them by a Hamiltonian isotopy (without crossing any walls) in order to create intersections. Namely, projecting $\mathbb{C}^{2}$ to $\mathbb{C}$ by the map $f(x, y)=x y$, $T_{1}$ and $T_{2}$ fiber above concentric circles $\gamma_{i}=\left\{|z-\epsilon|=r_{i}\right\}$, and inside each fiber they consist of the "equatorial" $S^{1}$-orbit where $|x|=|y|$. Deform $T_{1}$ by a Hamiltonian isotopy, without crossing $\epsilon$ or 0 , to a $S^{1}$-invariant Lagrangian torus $T_{1}^{\prime}$ which fibers above a closed curve $\gamma_{1}^{\prime}$ intersecting $\gamma_{2}$ in two points $p$ and $q$, and $T_{1}^{\prime}=f^{-1}\left(\gamma_{1}^{\prime}\right) \cap\{|x|=|y|\}$ (see Figure 6). Then $T_{1}^{\prime}$ and $T_{2}$ intersect along two circles, which can be handled either as a degenerate Morse-Bott type intersection $\left(C F^{*}\left(T_{1}^{\prime}, T_{2}\right)\right.$ is then generated by chains on $\left.T_{1}^{\prime} \cap T_{2}\right)$, or by further perturbing $T_{1}^{\prime}$ to replace each $S^{1}$ by two transverse intersection points.

Proposition 4.2. In $X^{0}=\mathbb{C}^{2} \backslash f^{-1}(\epsilon)$, the convergent power series Floer homology $H F^{*}\left(T_{1}^{\prime}, T_{2}\right)$ is well-defined and isomorphic to $H^{*}\left(T^{2}, \mathbb{C}\right)$.

Proof. Any holomorphic disc in $X^{0}=\mathbb{C}^{2} \backslash f^{-1}(\epsilon)$ that contributes to the Floer differential on $C F^{*}\left(T_{1}^{\prime}, T_{2}\right)$ is necessarily a section of $f$ over one of the two regions $R_{1}$ and $R_{2}$ delimited by $\gamma_{1}^{\prime}$ and $\gamma_{2}$ (see Figure 6 ). Recalling that $|x|=|y|$ on $T_{1}^{\prime} \cup T_{2}$, the maximum principle applied to $x / y$ implies that, if a disc with boundary in $T_{1}^{\prime} \cup T_{2}$ intersects neither the $x$ axis nor the $y$ axis, then $x / y$ is constant over it. Thus, there is exactly one $S^{1}$-family of such sections of $f$ over $R_{1}$, namely the portions of the lines $y=e^{i \varphi} x$ which lie in $f^{-1}\left(R_{1}\right)$. On the other hand, there are two $S^{1}$-families of sections over $R_{2}$. Indeed, let $g: D^{2} \rightarrow R_{2}$ be a biholomorphism given by the Riemann mapping theorem, chosen so that $g(0)=0$, and consider a holomorphic map $u:\left(D^{2}, \partial D^{2}\right) \rightarrow\left(X^{0}, T_{1}^{\prime} \cup T_{2}\right), z \mapsto u(z)=(x(z), y(z))$ such that $f \circ u$ maps 
$D^{2}$ biholomorphically onto $R_{2}$. Up to a reparametrization we can assume that $f \circ u=g$. Over the image of $u$, either $x$ or $y$ must vanish transversely once; assume that it is $x$ that vanishes. Then $z \mapsto x(z) / y(z)$ is a holomorphic function on the disc, taking values in the unit circle along the boundary, and vanishing once at the origin, therefore it is of the form $z \mapsto e^{i \varphi} z$ for some $e^{i \varphi} \in S^{1}$. Thus $u(z)=\left(e^{i \varphi / 2}(z g(z))^{1 / 2}, e^{-i \varphi / 2}(g(z) / z)^{1 / 2}\right)$. This gives an $S^{1}$ family of holomorphic sections over $R_{2}$; the other one is obtained similarly by exchanging $x$ and $y$.

Denote by $\alpha_{1}$ (resp. $\alpha_{2}$ ) the symplectic area of the holomorphic discs in $\left(X^{0}, T_{1}^{\prime} \cup T_{2}\right)$ which are sections of $f$ over $R_{1}$ (resp. $R_{2}$ ). By construction, these areas are related to those of the Maslov index 2 discs bounded by $T_{1}^{\prime}$ and $T_{2}$ in $\mathbb{C}^{2}$ : namely, $\alpha_{2}-\alpha_{1}=A_{2}-A_{1}$. Thus, the choices made above imply that $\exp \left(-\alpha_{1}\right)=2 \exp \left(-\alpha_{2}\right)$. After a careful check of signs, this in turn implies that the contributions of the various holomorphic discs in $\left(X^{0}, T_{1}^{\prime} \cup T_{2}\right)$ to the Floer differential on $C F^{*}\left(T_{1}^{\prime}, T_{2}\right)$ (with $\mathbb{C}$ coefficients) cancel out.

Denote by $e_{p}$ the generator of $C F^{0}\left(T_{1}^{\prime}, T_{2}\right)$ which comes from the intersections in $f^{-1}(p)$, and denote by $e_{q}$ the generator of $C F^{0}\left(T_{2}, T_{1}^{\prime}\right)$ which comes from the intersections in $f^{-1}(q)$. Then $\mathfrak{m}_{2}\left(e_{p}, e_{q}\right)=e^{-\alpha_{1}}\left[T_{1}^{\prime}\right]$ is a nonzero multiple of the unit in $C F^{*}\left(T_{1}^{\prime}, T_{1}^{\prime}\right)$, and $\mathfrak{m}_{2}\left(e_{q}, e_{p}\right)=e^{-\alpha_{1}}\left[T_{2}\right]$ is a nonzero multiple of the unit in $C F^{*}\left(T_{2}, T_{2}\right)$ : this makes it reasonable to state that $T_{1}^{\prime}$ and $T_{2}$ are isomorphic.

This example illustrates the failure of convergent power series Floer homology to be invariant under Hamiltonian isotopies, even without wallcrossing (recall the isotopy from $T_{1}$ to $T_{1}^{\prime}$ did not cross $f^{-1}(0)$ ); this is of course very different from the situation over the Novikov ring. When we deform $T_{1}^{\prime}$ back to $T_{1}$, we end up being able to cancel all the intersection points even though they represent nontrivial elements in Floer homology, because the cancellations in the Floer differential occur between families of discs with different symplectic areas (something which wouldn't be possible over Novikov coefficients). At the critical instant in the deformation, the discs with area $\alpha_{1}$ have shrunk to points, while the discs with area $\alpha_{2}$ become pinched annuli. At the end of the deformation, the tori $T_{1}$ and $T_{2}$ are disjoint, and the discs have become holomorphic annuli with boundary in $T_{1} \cup T_{2}$.

It would be tempting to hope that a souped up version of Floer theory that also includes holomorphic annuli would be better behaved. However, in that case we would immediately hit a divergence issue when working with complex coefficients: indeed, there are $2^{k}$ families of holomorphic annuli with boundary in $T_{1} \cup T_{2}$ which cover $k$-to- 1 the annulus bounded by the circles $\gamma_{1}$ and $\gamma_{2}$ in $\mathbb{C}$.

Even without considering annuli, divergence issues are already responsible for the bad properties of convergent power series Floer homology exhibited here - first and foremost, the lack of invariance under the Hamiltonian 
isotopy from $T_{1}$ to $T_{1}^{\prime}$. Denote by $H:[0,1] \times X^{0} \rightarrow \mathbb{R}$ a family of Hamiltonians whose time 1 flow sends $T_{1}$ to $T_{1}^{\prime}$, and recall that continuation maps $\Phi: C F^{*}\left(T_{1}, T_{2}\right) \rightarrow C F^{*}\left(T_{1}^{\prime}, T_{2}\right)$ and $\Phi: C F^{*}\left(T_{1}^{\prime}, T_{2}\right) \rightarrow C F^{*}\left(T_{1}, T_{2}\right)$ can be defined by counting index 0 solutions of perturbed holomorphic curve equations of the form

$$
\frac{\partial u}{\partial s}+J\left(\frac{\partial u}{\partial t}-\chi(s) X_{H}(t, u(s, t))\right)=0 .
$$

Here $u: \mathbb{R} \times[0,1] \rightarrow X^{0}$ is a map with $u(\mathbb{R} \times\{0\}) \subset T_{1}$ and $u(\mathbb{R} \times\{1\}) \subset T_{2}$ and satisfying suitable asymptotic conditions at infinity, $X_{H}$ is the Hamiltonian vector field associated to $H$, and $\chi: \mathbb{R} \rightarrow[0,1]$ is a suitable smooth cut-off function.

In our case, $\Phi$ and $\bar{\Phi}$ are obviously zero since $C F^{*}\left(T_{1}, T_{2}\right)=0$; this of course prevents $\Phi \circ \bar{\Phi}: C F^{*}\left(T_{1}^{\prime}, T_{2}\right) \rightarrow C F^{*}\left(T_{1}^{\prime}, T_{2}\right)$ from being homotopic to identity as expected. Specifically, the homotopy would normally be constructed by considering exceptional index -1 solutions to (4.4) where the cut-off $\chi$ is equal to 1 near $\pm \infty$ and $\int_{\mathbb{R}}(1-\chi)$ varies between 0 and infinity. In the present case, a calculation shows that that there are infinitely many exceptional solutions - in fact there are $2^{k}$ solutions of energy $k\left(\alpha_{2}-\alpha_{1}\right)$ for each integer $k$, which makes the homotopy divergent. (To see this, choose the Hamiltonian isotopy from $T_{1}$ to $T_{1}^{\prime}$ to be lifted from the complex plane by the projection $f$, and look at similar continuation maps between $C F^{*}\left(\gamma_{1}, \gamma_{2}\right)=0$ and $C F^{*}\left(\gamma_{1}^{\prime}, \gamma_{2}\right)$ inside $\mathbb{C} \backslash\{\epsilon\}$. In that case, an explicit calculation shows that there is an infinite sequence of exceptional index -1 solutions to (4.4), wrapping once, twice, etc. around the annulus bounded by $\gamma_{1}$ and $\gamma_{2}$. Moreover, the exceptional trajectory which wraps $k$ times around the annulus in $\mathbb{C} \backslash\{\epsilon\}$ can be shown to admit $2^{k} S^{1}$-families of lifts to $X^{0}$.)

Another instance of divergence occurs if we try to test the associativity of the product in Floer homology. Namely, in addition to the isomorphisms $e_{p} \in C F^{0}\left(T_{1}^{\prime}, T_{2}\right)$ and $e_{q} \in C F^{0}\left(T_{2}, T_{1}^{\prime}\right)$ considered above, denote by $e_{a} \in C F^{0}\left(T_{1}^{\prime}, T_{1}\right)$, resp. $e_{b} \in C F^{0}\left(T_{1}, T_{1}^{\prime}\right)$, the generators which come from the intersections in $f^{-1}(a)$, resp. $f^{-1}(b)$ (see Figure 7 ). One easily checks that $\mathfrak{m}_{2}\left(e_{a}, e_{b}\right)$ is a nonzero multiple of the unit in $C F^{*}\left(T_{1}^{\prime}, T_{1}^{\prime}\right)$. Then we can try to compose $e_{a}, e_{b}$ and $e_{p}$ in two different ways: $\mathfrak{m}_{2}\left(\mathfrak{m}_{2}\left(e_{a}, e_{b}\right), e_{p}\right)$ is

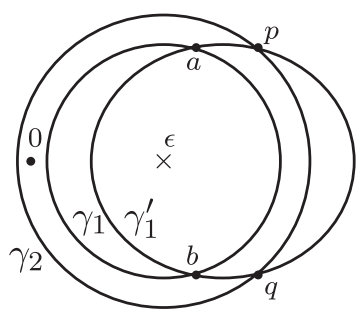

Figure $7 . T_{1}, T_{1}^{\prime}$ and $T_{2}$ 
a nonzero multiple of $e_{p}$, whereas $\mathfrak{m}_{2}\left(e_{a}, \mathfrak{m}_{2}\left(e_{b}, e_{p}\right)\right)$ is zero since $\mathfrak{m}_{2}\left(e_{b}, e_{p}\right) \in$ $C F^{*}\left(T_{1}, T_{2}\right)=0$. Passing to cohomology classes, this contradicts the expected associativity of the product on Floer homology. A closer inspection reveals that this is caused by the divergence of quantities such as $\mathfrak{m}_{3}\left(e_{a}, f_{a}, e_{p}\right)$ (where $f_{a}$ is the generator of $C F^{1}\left(T_{1}, T_{1}^{\prime}\right)$ corresponding to the intersections in $f^{-1}(a)$ ): indeed, this triple product counts discs obtained by cutting open the divergent series of annuli with boundary in $T_{1} \cup T_{2}$ already mentioned above.

In conclusion, there are many pitfalls associated to the use of convergent power series Floer homology, even in fairly simple situations (compactifying the above example to $\mathbb{C P}^{2}$, we would still encounter the same divergence phenomena in a smooth projective Fano variety). A cautious view of the situation would dictate that outside of the very simplest cases it is illusory to even attempt to work over complex coefficients, and that in general mirror symmetry is only a perturbative phenomenon taking place over a formal neighborhood of the large volume limit. Nonetheless, as long as one restricts oneself to consider only certain aspects of Floer theory, the power series obtained by working over the Novikov ring seem to often have good enough convergence properties to allow the construction of a mirror that is an honest complex manifold (rather than a scheme over the Novikov field). Floer theory for a single weakly unobstructed Lagrangian seems to be less prone to divergence than the theory for pairs such as $\left(L_{1}, L_{2}\right)$ in the above example. Also, in the example we have considered, divergence issues can be avoided by equipping all our Lagrangian submanifolds with suitable Hamiltonian perturbation data (i.e., "wiggling" Lagrangians so that they intersect sufficiently). However, more sophisticated divergent examples can be built e.g. inside conic bundles over elliptic curves; in some of these examples, Floer products are given by series in $\Lambda_{0}$ for which the radius of convergence is strictly less than 1, i.e. convergence only holds for sufficiently large symplectic forms, regardless of Hamiltonian perturbations.

\section{Relative mirror symmetry}

5.1. Mirror symmetry for pairs. In this section, we turn to mirror symmetry for a pair $(X, D)$, where $X$ is a Kähler manifold and $D$ is a smooth Calabi-Yau hypersurface in the anticanonical linear system. Our goal is to clarify the folklore statement that "the fiber of the mirror superpotential $W$ : $X^{\vee} \rightarrow \mathbb{C}$ is mirror to $D$ ". The discussion is fairly similar to that in $\S 7$ of [4].

Let $D \subset X$ be a hypersurface in the anticanonical linear system, with defining section $\sigma \in H^{0}\left(X, K_{X}^{-1}\right)$ : then the holomorphic volume form $\Omega=$ $\sigma^{-1} \in \Omega^{n, 0}(X \backslash D)$ (with poles along $D$ ) induces a holomorphic volume form $\Omega_{D}$ on $D$, the residue of $\Omega$ along $D$, characterized by the property that $\Omega=\Omega_{D} \wedge d \log \sigma+O(1)$ in a neighborhood of $D$. Additionally, the Kähler form $\omega$ induces a Kähler form on $D$ by restriction. 
It is reasonable to expect that special Lagrangian torus fibrations on $X \backslash D$ should have a "nice" boundary behavior. Namely, assuming that the Kähler metric on $X$ is complete, for a reasonable special Lagrangian fibration $\pi: X \backslash D \rightarrow B$ we expect:

ConjeCture 5.1. Near $\partial B$, the fibers of $\pi$ are contained in a neighborhood of $D$, and the smooth fibers are $S^{1}$-bundles over special Lagrangian tori in $\left(D, \omega_{\mid D}, \Omega_{D}\right)$.

(Here, by $\partial B$ we mean the part of the boundary of $B$ which lies at finite distance in the symplectic affine structure).

In other terms, we expect that near $D$ the special Lagrangian tori in $X \backslash D$ accumulate onto special Lagrangian tori in $D$ (as observed in the various examples we have discussed). If Conjecture 5.1 holds, then $\partial B$ is the base of a special Lagrangian fibration on $D$, and the (uncorrected) SYZ mirror to $D, M_{D}$, can be identified as a complex hypersurface lying inside the boundary of the (uncorrected) moduli space $M$ of pairs $(L, \nabla)$ in $X \backslash D$.

Assume $D$ is smooth, and consider a special Lagrangian torus fiber $L=\pi^{-1}(b)$ near $\partial B$ : then we expect that $L$ bounds a distinguished family of Maslov index 2 holomorphic discs, namely small meridian discs in the normal direction to $D$. More precisely, as $b$ approaches the boundary of $B$, we expect $L$ to collapse onto a special Lagrangian torus $\Lambda$ in $D$, and the meridian discs to be approximated by small discs inside the fibers of the normal bundle of $D$ lying above the points of $\Lambda$.

Call $\delta$ the relative homotopy class of the meridian discs, and by $z_{\delta}$ the corresponding holomorphic coordinate on $M$ (which is also the contribution of the family of meridian discs to the superpotential). Then we expect that $z_{\delta}$ is the dominant term in the superpotential near the boundary of $M$, as the meridian discs have areas tending to zero and all the other holomorphic discs have comparatively much greater areas.

The boundary of $M$ corresponds to limiting pairs $(L, \nabla)$ where the area of the meridian disc becomes 0 (i.e., $L$ is entirely collapsed onto a special Lagrangian torus in $D)$; recalling that $\left|z_{\delta}\right|=\exp \left(-\int_{\delta} \omega\right)$, this corresponds to $\left|z_{\delta}\right|=1$. In fact, the boundary of $M$ fibers above the unit circle, via the map

$$
\arg \left(z_{\delta}\right): \partial M=\left\{\left|z_{\delta}\right|=1\right\} \rightarrow S^{1},
$$

with fiber $M_{D}=\left\{z_{\delta}=1\right\}$. The points of $M_{D}$ correspond to pairs $(L, \nabla)$ where $L$ is entirely collapsed onto a special Lagrangian torus $\Lambda \subset D$, and the holonomy of $\nabla$ around the meridian loop $\mu=\partial \delta$ is trivial, i.e. $\nabla$ is pulled back from a $U(1)$ local system on $\Lambda$. Thus $M_{D}$ is precisely the uncorrected SYZ mirror to $D$.

In general, the fibration (5.1) has monodromy. Indeed, a local trivialization is given by fixing a framing, i.e. an $(n-1)$-dimensional subspace of $H_{1}(L, \mathbb{Z})$ which under the projection $L \rightarrow \Lambda$ maps isomorphically onto $H_{1}(\Lambda, \mathbb{Z})$. (Less intrinsically, we can choose a set of longitudes, i.e. lifts to $L$ 
of a collection of $n-1$ loops generating $H_{1}(\Lambda, \mathbb{Z})$ ); the framing data allows us to lift to $M$ a set of local holomorphic coordinates on $M_{D}$. However, unless the normal bundle to $D$ is trivial there is no consistent global choice of framings: if we move $\Lambda$ around a loop in $\partial B$ and keep track of a longitude $\lambda$ lifting a loop $\gamma \in \Lambda$, the monodromy action is of the form $\lambda \mapsto \lambda+k_{\gamma} \mu$, where $k_{\gamma}$ is the degree of the normal bundle of $D$ over the surface traced out by $\gamma$.

A more thorough calculation shows that the monodromy of (5.1) is given by a symplectomorphism of $M_{D}$ which geometrically realizes (as a fiberwise translation in the special Lagrangian fibration $M_{D} \rightarrow \partial B$ dual to the SYZ fibration on $D$ ) the mirror to the autoequivalence $-\otimes K_{X \mid D}^{-1}$ of $D^{b} \operatorname{Coh}(D)$.

This is easiest to see if we assume that, in a neighborhood of $D$, the anticanonical bundle $K_{X}^{-1}$ can be equipped with a semi-flat connection, i.e. a holomorphic connection whose restriction to the fibers of $\pi$ is flat. Then the parallel transport from one fiber of (5.1) to another can be realized geometrically as follows: given a pair $(L, \nabla)$ where $L$ is almost collapsed onto a special Lagrangian $\Lambda \subset D$, we can modify the holonomy of $\nabla$ around the meridian loop by adding to it a multiple of $\operatorname{Im}\left(\sigma^{-1} \partial \sigma\right)_{\mid L}$, where $\sigma$ is the defining section of $D$. The monodromy is then $(L, \nabla) \mapsto\left(L, \nabla+\operatorname{Im}\left(\sigma^{-1} \partial \sigma\right)_{\mid L}\right)$, which in the limit where $L$ collapses onto $D$ is exactly the expected transformation.

If we can neglect the terms other than $z_{\delta}$ in the superpotential, for instance in the large volume limit, then $M_{D}$ is essentially identified with the fiber of $W$ at 1 . In fact, recall from the discussion at the end of $\S 2.2$ that changing the Kähler class to $[\omega]+t c_{1}(X)$ "enlarges" the mirror while rescaling the superpotential by a factor of $e^{-t}$ : thus, assuming that $X$ is Fano, or more generally that $-K_{X}$ is nef, the flow to the large volume limit can be realized simply by rescaling the superpotential. Hence, Conjecture 5.1 implies:

Conjecture 5.2. If $\left(X^{\vee}, W\right)$ is mirror to $X$, and if $-K_{X}$ is nef, then for $t \rightarrow \infty$ the family of hypersurfaces $\left\{W=e^{t}\right\} \subset X^{\vee}$ is asymptotic (up to corrections that decrease exponentially with $t$ ) to the family of mirrors to $\left(D, \omega_{\mid D}+t c_{1}(X)_{\mid D}\right)$.

For example, considering the mirror to $\mathbb{C P}^{2}$ with $[\omega] \cdot\left[\mathbb{C P}^{1}\right]=\Lambda$, the $j$-invariant of the elliptic curve $\left\{x+y+e^{-\Lambda} / x y=e^{t}\right\} \subset\left(\mathbb{C}^{*}\right)^{2}$ can be determined to equal

$$
\frac{e^{3 t+\Lambda}\left(e^{3 t+\Lambda}-24\right)^{3}}{e^{3 t+\Lambda}-27}=e^{9 t+3 \Lambda}+\cdots
$$

whose leading term matches with the symplectic area of the anticanonical divisor after inflation (observe that $\left([\omega]+t c_{1}\right) \cdot\left[\mathbb{C P}^{1}\right]=3 t+\Lambda$ ).

There are two reasons why this statement only holds asymptotically for $t \rightarrow \infty$. First, the formula for the superpotential includes other terms besides $z_{\delta}$, so the hypersurfaces $\left\{W=e^{t}\right\}$ and $\left\{z_{\delta}=e^{t}\right\}$ are not quite the same. 
More importantly, the instanton corrections to the mirror of $D$ are not the same as the instanton corrections to the fiber of $z_{\delta}$. When constructing the mirror to $X$, the geometry of $M_{D} \subset M$ gets corrected by wall-crossing terms that record holomorphic Maslov index 0 discs in $X$; whereas, when constructing the mirror of $D$, the corrections only arise from Maslov index 0 holomorphic discs in $D$.

In other terms: the instanton corrections to the mirror of $X$ arise from walls generated by singularities in the fibration $\pi: X \backslash D \rightarrow B$ (i.e., singularities in the affine structure of $B$ ), whereas the instanton corrections to the mirror of $D$ arise from the walls generated by singularities in the fibration $\pi_{D}: D \rightarrow \partial B$ (i.e., singularities in the affine structure of $\partial B$ ). Since the singularities of the affine structure on $\partial B$ are induced by those strata of singularities of $B$ that hit the boundary, the wall-crossing phenomena in $D$ are induced by a subset of the wall-crossing phenomena in $X$, but there are also walls in $X$ which hit the boundary of $B$ without being induced by singularities at the boundary.

On the other hand, the smooth fibers of $W$ are symplectomorphic to each other and to the hypersurface $\left\{z_{\delta}=1\right\}$. Moreover, it is generally believed that the Kähler class of the mirror should not be affected by instanton corrections, so the discrepancy discussed above is no longer an issue. Hence: we expect that the fibers of $W$, viewed as symplectic manifolds, are mirror to the divisor $D$ viewed as a complex manifold. (Observe that, from this perspective, the parameter $t$ in Conjecture 5.2 no longer plays any role, and accordingly the geometries are expected to match on the nose.)

5.2. Homological mirror symmetry. Assuming Conjectures 5.1 and 5.2 , we can try to compare the statements of homological mirror symmetry for $X$ and for the Calabi-Yau hypersurface $D$. Due to the mismatch between the complex structure on the mirror to $D$ and that on the fibers of $W$ (see Conjecture 5.2), in general we can only hope to achieve this in one direction, namely relating the derived categories of coherent sheaves on $X$ and $D$ with the Fukaya categories of their mirrors.

Denote by $\left(X^{\vee}, W\right)$ the mirror to $X$, and by $D^{\vee}$ the mirror to $D$, which we identify symplectically with a fiber of $W$, say $D^{\vee}=\left\{W=e^{t}\right\} \subset X^{\vee}$ for fixed $t \gg 0$.

First we need to briefly describe the Fukaya category of the LandauGinzburg model $W: X^{\vee} \rightarrow \mathbb{C}$. The general idea, which goes back to Kontsevich [27] and Hori-Iqbal-Vafa [22], is to allow as objects admissible Lagrangian submanifolds of $X^{\vee}$; these can be described either as potentially non-compact Lagrangian submanifolds which, outside of a compact subset, are invariant under the gradient flow of $-\operatorname{Re}(W)$, or, truncating, as compact Lagrangian submanifolds with (possibly empty) boundary contained inside a fixed reference fiber of $W$ (and satisfying an additional condition). The case of Lefschetz fibrations (i.e., when the critical points of $W$ are nondegenerate) has been studied in great detail by Seidel; in this case, which is 
by far the best understood, the theory can be formulated in terms of the vanishing cycles at the critical points (see e.g. [35]).

The formulation which is the most relevant to us is the one discussed by Abouzaid in [1]]: in this version, one considers Lagrangian submanifolds of $X^{\vee}$ with boundary contained in the given reference fiber $D^{\vee}=W^{-1}\left(e^{t}\right)$, and which near the reference fiber are mapped by $W$ to an embedded curve $\gamma \subset \mathbb{C}$.

Definition 5.3. A Lagrangian submanifold $L \subset X^{\vee}$ with (possibly empty) boundary $\partial L \subset D^{\vee}=W^{-1}\left(e^{t}\right)$ is admissible with phase $\varphi \in\left(-\frac{\pi}{2}, \frac{\pi}{2}\right)$ if $|W|<e^{t}$ at every point of $\operatorname{int}(L)$ and, near $\partial L$, the restriction of $W$ to $L$ takes values in the half-line $e^{t}-e^{i \varphi} \mathbb{R}_{+}$.

Floer theory is then defined by choosing a specific set of Hamiltonian perturbations, which amounts to deforming the given admissible Lagrangians so that their phases are in increasing order, and ignoring boundary intersections. For instance, to determine $H F\left(L_{1}, L_{2}\right)$, one first deforms $L_{2}$ (rel. its boundary) to an admissible Lagrangian $L_{2}^{+}$whose phase is greater than that of $L_{1}$, and one computes Floer homology for the pair of Lagrangians $\left(L_{1}, L_{2}^{+}\right)$ inside $X^{\vee}$, ignoring boundary intersections. We denote by $\mathcal{F}\left(X^{\vee}, D^{\vee}\right)$ the Fukaya category constructed in this manner. (In fact, strictly speaking, one should place the reference fiber "at infinity", i.e. either consider a limit of this construction as $t \rightarrow+\infty$, or enlarge the symplectic structure on the subset $\left\{|W|<e^{t}\right\}$ of $X^{\vee}$ so that the symplectic form blows up near the boundary and the Kähler metric becomes complete; for simplicity we ignore this subtlety.)

By construction, the boundary of an admissible Lagrangian in $X^{\vee}$ is a Lagrangian submanifold of $D^{\vee}$ (possibly empty, and not necessarily connected). There is a restriction $A_{\infty}$-functor $\rho: \mathcal{F}\left(X^{\vee}, D^{\vee}\right) \rightarrow \mathcal{F}\left(D^{\vee}\right)$ from the Fukaya category of the Landau-Ginzburg model $\left(X^{\vee}, W\right)$ to the (usual) Fukaya category of $D^{\vee}$. At the level of objects, this is simply $(L, \nabla) \mapsto$ $\left(\partial L, \nabla_{\mid \partial L}\right)$. At the level of morphisms, the $A_{\infty}$-functor $\rho$ consists of a collection of maps

$$
\begin{aligned}
\rho_{(k)} & : \operatorname{Hom}_{\mathcal{F}\left(X^{\vee}, D^{\vee}\right)}\left(L_{1}, L_{2}\right) \otimes \cdots \otimes \operatorname{Hom}_{\mathcal{F}\left(X^{\vee}, D^{\vee}\right)}\left(L_{k}, L_{k+1}\right) \\
& \rightarrow \operatorname{Hom}_{\mathcal{F}\left(D^{\vee}\right)}\left(\partial L_{1}, \partial L_{k+1}\right) .
\end{aligned}
$$

The first order term $\rho_{(1)}$ is the easiest to describe: given an intersection point $p \in \operatorname{int}\left(L_{1}\right) \cap \operatorname{int}\left(L_{2}^{+}\right), \rho_{(1)}(p)$ is a linear combination of intersection points in which the coefficient of $q \in \partial L_{1} \cap \partial L_{2}$ counts holomorphic strips in $\left(X^{\vee}, L_{1} \cup L_{2}^{+}\right)$connecting $p$ to $q$. Similarly, given $k+1$ admissible Lagrangians $L_{1}, \ldots, L_{k+1}$, and perturbing them so that their phases are in increasing order, $\rho_{(k)}$ counts holomorphic discs in $\left(X^{\vee}, \bigcup L_{i}^{+}\right)$with $k$ corners at prescribed interior intersection points and one corner at a boundary intersection point. 
Homological mirror symmetry for the pair $(X, D)$ can then be summarized by the following conjecture:

CONJeCTURE 5.4. There is a commutative diagram

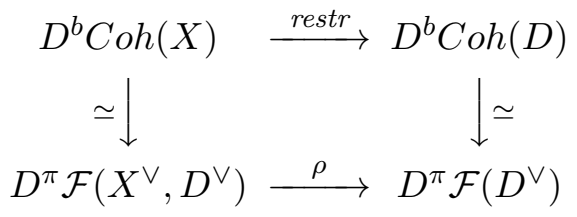

In this diagram, the horizontal arrows are the restriction functors, and the vertical arrows are the equivalences predicted by homological mirror symmetry. The reader is referred to $[\mathbf{6}]$ for a verification in the case of Del Pezzo surfaces.

Another type of Fukaya category that can be associated to $X^{\vee}$ is its wrapped Fukaya category $\mathcal{F}_{w r}\left(X^{\vee}\right)[\mathbf{3}]$. The objects of that category are again non-compact Lagrangian submanifolds, but the Hamiltonian perturbations used to define Floer homology now diverge at infinity. Assuming that $W$ is proper, we can e.g. use the Hamiltonian flow generated by a function of $|W|$ that increases sufficiently quickly at infinity; however, the wrapped category can be defined purely in terms of the symplectic geometry of $X^{\vee}$ at infinity, without reference to the superpotential (see [3]).

Homological mirror symmetry for the open Calabi-Yau $X \backslash D$ then predicts an equivalence between the derived category of coherent sheaves $D^{b} \operatorname{Coh}(X \backslash D)$ and the derived wrapped Fukaya category $D^{\pi} \mathcal{F}_{w r}\left(X^{\vee}\right)$. Moreover, the restriction functor from $D^{b} \operatorname{Coh}(X)$ to $D^{b} \operatorname{Coh}(X \backslash D)$ is expected to correspond to a natural functor $\varpi$ from the Fukaya category of the Landau-Ginzburg model $\left(X^{\vee}, W\right)$ to the wrapped Fukaya category of $X^{\vee}$. On objects, $\varpi$ is essentially identity (after sending the reference fiber to infinity, or extending admissible Lagrangians to non-compact ones by parallel transport along the gradient flow of $\operatorname{Re}(W)$ ). On morphisms, $\varpi$ is essentially an inclusion map if we set up the Hamiltonian perturbations in the wrapped category to be supported outside of the region where $|W|<e^{t}$; or, more intrinsically, $\varpi$ is the continuation map induced on Floer complexes by the deformation from the small Hamiltonian perturbations used to define the Fukaya category of $\left(X^{\vee}, W\right)$ to the large Hamiltonian perturbations used to define the wrapped category.

In fact, the wrapped Fukaya category can alternatively be defined from $\mathcal{F}\left(X^{\vee}, W\right)$ as the result of localization with respect to a certain natural transformation from the Serre functor (up to a shift) to the identity, induced by the monodromy of $W$ near infinity (see $\S 4$ of [36] and $\S 6$ of [37]); this parallels the fact that $D^{b} \operatorname{Coh}(X \backslash D)$ is the localization of $D^{b} \operatorname{Coh}(X)$ with respect to the natural transformation from $-\otimes K_{X}$ (i.e., the Serre functor up to a shift) to the identity given by the defining section of $D$. 
Finally, when considering compact closed Lagrangian submanifolds, there is no difference between the Fukaya category of $\left(X^{\vee}, W\right)$ and the wrapped Fulaya category; the full subcategory consisting of these compact objects is expected to be equivalent to the subcategory of $D^{b} \operatorname{Coh}(X \backslash D)$ generated by complexes with compactly supported cohomology.

5.3. Complete intersections. As pointed out to the author by Ludmil Katzarkov, the above ideas can be extended to understand mirror symmetry for complete intersections (remaining in the framework of manifolds with effective anticanonical divisors). Namely, consider divisors $D_{1}, \ldots, D_{k} \subset X$ (smooth, or at most with normal crossing singularities), intersecting each other transversely, such that $\sum D_{i}=-K_{X}$. Let $\left(X^{\vee}, W\right)$ be the mirror of $X$ relative to the anticanonical divisor $\sum D_{i}$ : then the superpotential on $X^{\vee}$ splits into a sum $W=W_{1}+\cdots+W_{k}$, where $W_{i}$ : $X^{\vee} \rightarrow \mathbb{C}$ records the contributions to the superpotential of holomorphic Maslov index 2 discs which hit the component $D_{i}$ of the anticanonical divisor.

For a subset $I \subseteq\{1, \ldots, k\}$, consider the complete intersection $X_{I}=$ $\bigcap_{i \in I} D_{i} \subset X$, and the divisors $D_{I, j}=X_{I} \cap D_{j}, j \notin I$, whose sum represents the anticanonical class of $X_{I}$. Then we have:

Conjecture 5.5. In the large volume limit $t \rightarrow \infty$, the mirror to $X_{I}$ equipped with the Kähler form $\omega_{\mid X_{I}}+t c_{1}(X)_{\mid X_{I}}$ and the anticanonical divisor $\sum_{j \notin I} D_{I, j}$ is approximated (in the sense of Conjecture 5.2) by the complete intersection $X_{I}^{\vee}:=\bigcap_{i \in I} W_{i}^{-1}\left(e^{t}\right)$ in $X^{\vee}$, equipped with the superpotential $W_{I}:=\sum_{j \notin I} W_{j}$.

As before, if we are only interested in comparing the complex geometry of $X_{I}$ with the symplectic geometry of $\left(X_{I}^{\vee}, W_{I}\right)$, then the construction does not depend on the parameter $t$, and passage to the large volume limit is not needed.

Conjecture 5.5 can be understood geometrically as follows. In this setting, we expect to have a special Lagrangian torus fibration $\pi: X \backslash\left(\bigcup D_{i}\right) \rightarrow$ $B$, whose base $B$ has boundary and corners: at the boundary, the special Lagrangian fibers collapse onto one of the hypersurfaces $D_{i}$, and at the corners they collapse onto the intersection of several $D_{i}$. (This picture is e.g. obvious in the toric setting, where $B$ is the interior of the moment polytope.)

Whenever the fibers of $\pi$ lie sufficiently close to $D_{i}$, they are expected to bound small meridian discs intersecting $D_{i}$ transversely once, whereas the other families of discs have comparatively larger symplectic area, so that $W_{i}=z_{\delta_{i}}+o(1)$. Setting $z_{\delta_{i}}$ equal to 1 for $i \in I$ amounts to considering special Lagrangian tori that are completely collapsed onto $X_{I}=\cap_{i \in I} D_{i}$, equipped with flat connections that have trivial holonomy along the meridian loops, i.e. are pulled back from special Lagrangian tori in $X_{I}$. Thus, before instanton corrections, $\bigcap_{i \in I}\left\{z_{\delta_{i}}=1\right\}$ is the (uncorrected) SYZ mirror 
to $X_{I} \backslash\left(\bigcup_{j \notin I} D_{I, j}\right)$. When $t \rightarrow \infty$ the discrepancy between $W_{i}$ and $z_{\delta_{i}}$ and the differences in instanton corrections are expected to become negligible.

Moreover, in the limit where $L \subset X \backslash\left(\bigcup D_{i}\right)$ collapses onto a special Lagrangian $\Lambda \subset X_{I} \backslash\left(\bigcup_{j \notin I} D_{I, j}\right)$, for $j \notin I$ the dominant terms in $W_{j}$ should correspond to families of holomorphic discs in $(X, L)$ that converge to holomorphic discs in $\left(X_{I}, \Lambda\right)$ (intersecting $\left.D_{I, j}\right)$. Hence, $\sum_{j \notin I} W_{j}$ should differ from the superpotential for the mirror to $X_{I}$ by terms that become negligible in the large volume limit.

As a special case of Conjecture 5.5, taking $I=\{1, \ldots, k\}$, (in the large volume limit) the fiber of $\left(W_{1}, \ldots, W_{k}\right)$ is mirror to the Calabi-Yau complete intersection $X_{\{1, \ldots, k\}}=D_{1} \cap \cdots \cap D_{k}$. (In this case there is no residual superpotential.) This is consistent with standard conjectures.

It is also worth noting that, in a degenerate toric limit, Conjecture 5.5 recovers the predictions made by Hori and Vafa $[\mathbf{2 3}]$ for mirrors of Fano complete intersections in toric varieties. To give a simple example, consider $X=\mathbb{C P}^{3}$ (with $\int_{\mathbb{C P}^{1}} \omega=\Lambda$ ), and let $D_{1}, D_{2} \subset X$ be quadric surfaces intersecting transversely in an elliptic curve $E=D_{1} \cap D_{2}$. Then the superpotential on $X^{\vee}$ decomposes as a sum $W=W_{1}+W_{2}$. In the degenerate limit where $D_{1}$ and $D_{2}$ are toric quadrics consisting of two coordinate hyperplanes each, and $E$ is a singular elliptic curve with four rational components, we have $X^{\vee}=\left\{z_{0} z_{1} z_{2} z_{3}=e^{-\Lambda}\right\} \subset\left(\mathbb{C}^{*}\right)^{4}$, and $W=W_{1}+W_{2}$, where $W_{1}=z_{0}+z_{1}$ and $W_{2}=z_{2}+z_{3}$. Then the mirror to $D_{1}$ is the surface

$$
\left\{z_{0} z_{1} z_{2} z_{3}=e^{-\Lambda}, z_{0}+z_{1}=e^{t}\right\} \subset\left(\mathbb{C}^{*}\right)^{4},
$$

equipped with the superpotential $W_{2}=z_{2}+z_{3}$, and similarly for $D_{2}$; and the mirror to $E$ is the curve $\left\{z_{0} z_{1} z_{2} z_{3}=e^{-\Lambda}, z_{0}+z_{1}=e^{t}, z_{2}+z_{3}=e^{t}\right\}$ (a noncompact elliptic curve with four punctures). These formulas are essentially identical to those in Hori-Vafa [23]. To be more precise: viewing $D_{i}$ and $E$ as symplectic manifolds (in which case the degeneration to the toric setting should be essentially irrelevant, i.e. up to a fiberwise compactification of the Landau-Ginzburg models we can think of smooth quadrics and elliptic curves), but taking the large volume limit $t \rightarrow \infty$, these formulas give an approximation to the complex geometry of the mirrors. On the other hand, if we consider the symplectic geometry of the mirrors, then the formulas give exact mirrors to $D_{i}$ and $E$ viewed as singular complex manifolds (torically degenerated quadrics and elliptic curves, i.e. large complex structure limits). Thus Hori and Vafa's formulas for toric complete intersections should be understood as a construction of the mirror at a limit point in both the complex and Kähler moduli spaces.

\section{References}

[1] M. Abouzaid, Homogeneous coordinate rings and mirror symmetry for toric varieties, Geom. Topol. 10 (2006), 1097-1157.

[2] M. Abouzaid, D. Auroux, L. Katzarkov, in preparation. 
[3] M. Abouzaid, P. Seidel, An open string analogue of Viterbo functoriality, arXiv:0712.3177.

[4] D. Auroux, Mirror symmetry and T-duality in the complement of an anticanonical divisor, J. Gökova Geom. Topol. 1 (2007), 51-91 (arXiv:0706.3207).

[5] D. Auroux, L. Katzarkov, D. Orlov, Mirror symmetry for weighted projective planes and their noncommutative deformations, Ann. Math. 167 (2008), 867-943.

[6] D. Auroux, L. Katzarkov, D. Orlov, Mirror symmetry for Del Pezzo surfaces: Vanishing cycles and coherent sheaves, Inventiones Math. 166 (2006), 537-582.

[7] A. Bondal, D. Orlov, Derived categories of coherent sheaves, Proc. International Congress of Mathematicians, Vol. II (Beijing, 2002), Higher Ed. Press, Beijing, 2002, pp. 47-56 (math.AG/0206295).

[8] P. Candelas, X. C. De La Ossa, P. S. Green, L. Parkes, A pair of Calabi-Yau manifolds as an exactly soluble superconformal theory, Nucl. Phys. B 359 (1991), 21.

[9] C.-H. Cho, Products of Floer cohomology of torus fibers in toric Fano manifolds, Comm. Math. Phys. 260 (2005), 613-640 (math.SG/0412414).

[10] C.-H. Cho, Non-displaceable Lagrangian submanifolds and Floer cohomology with non-unitary line bundle, arXiv:0710.5454.

[11] C.-H. Cho, Y.-G. Oh, Floer cohomology and disc instantons of Lagrangian torus fibers in Fano toric manifolds, Asian J. Math. 10 (2006), 773-814 (math.SG/0308225).

[12] O. Cornea, F. Lalonde, Cluster homology, math.SG/0508345.

[13] D. A. Cox, S. Katz, Mirror symmetry and algebraic geometry, Math. Surveys Monographs 68, Amer. Math. Soc., Providence, 1999.

[14] K. Fukaya, Y.-G. Oh, H. Ohta, K. Ono, Lagrangian intersection Floer theory: anomaly and obstruction, expanded version, 2006.

[15] K. Fukaya, Y.-G. Oh, H. Ohta, K. Ono, Lagrangian Floer theory on compact toric manifolds $I$, arXiv:0802.1703.

[16] M. Gross, Topological mirror symmetry, Inventiones Math. 144 (2001), 75-137.

[17] M. Gross, Special Lagrangian Fibrations II: Geometry, Winter School on Mirror Symmetry, Vector Bundles and Lagrangian Submanifolds (Cambridge, MA, 1999), AMS/IP Stud. Adv. Math. 23, Amer. Math. Soc., Providence, 2001, pp. 95-150 (math.AG/9809072).

[18] M. Gross, B. Siebert, From real affine geometry to complex geometry, math.AG/0703822.

[19] M. Gross, B. Siebert, An invitation to toric degenerations, arXiv:0808.2749.

[20] N. Hitchin, The moduli space of special Lagrangian submanifolds, Ann. Scuola Norm. Sup. Pisa Cl. Sci. 25 (1997), 503-515.

[21] K. Hori, Mirror symmetry and quantum geometry, Proc. ICM (Beijing, 2002), Higher Ed. Press, Beijing, 2002, vol. III, 431-443 (hep-th/0207068).

[22] K. Hori, A. Iqbal, C. Vafa, D-branes and mirror symmetry, hep-th/0005247.

[23] K. Hori, C. Vafa, Mirror symmetry, hep-th/0002222.

[24] D. Joyce, Lectures on Calabi-Yau and special Lagrangian geometry, math. DG/0108088.

[25] A. Kapustin, Y. Li, D-branes in Landau-Ginzburg models and algebraic geometry, J. High Energy Phys. 0312 (2003), 005 (hep-th/0210296).

[26] M. Kontsevich, Homological algebra of mirror symmetry, Proc. International Congress of Mathematicians (Zürich, 1994), Birkhäuser, Basel, 1995, pp. 120-139.

[27] M. Kontsevich, Lectures at ENS, Paris, Spring 1998, notes taken by J. Bellaiche, J.-F. Dat, I. Marin, G. Racinet and H. Randriambololona.

[28] M. Kontsevich, Y. Soibelman, Homological mirror symmetry and torus fibrations, Symplectic geometry and mirror symmetry (Seoul, 2000), World Sci. Publ., 2001, pp. 203-263 (math.SG/0011041). 
[29] M. Kontsevich, Y. Soibelman, Affine structures and non-Archimedean analytic spaces, The unity of mathematics, Progr. Math. 244, Birkhäuser Boston, 2006, pp. 321-385 (math.AG/ 0406564).

[30] N.C. Leung, Mirror symmetry without corrections, math.DG/0009235.

[31] C. C. Liu, Moduli of J-holomorphic curves with Lagrangian boundary conditions and open Gromov-Witten invariants for an $S^{1}$-equivariant pair, math.SG/0210257.

[32] R. C. McLean, Deformations of calibrated submanifolds, Comm. Anal. Geom. 6 (1998), 705-747.

[33] D. Orlov, Triangulated categories of singularities and D-branes in Landau-Ginzburg models, Proc. Steklov Inst. Math. 246 (2004), 227-248 (math.AG/0302304).

[34] P. Seidel, Fukaya categories and deformations, Proc. International Congress of Mathematicians, Vol. II (Beijing, 2002), Higher Ed. Press, Beijing, 2002, pp. 351-360.

[35] P. Seidel, Fukaya categories and Picard-Lefschetz theory, Zurich Lect. in Adv. Math., European Math. Soc., Zürich, 2008.

[36] P. Seidel, Symplectic homology as Hochschild homology, math.SG/0609037.

[37] P. Seidel, $A_{\infty}$-subalgebras and natural transformations, Homology, Homotopy Appl. 10 (2008), 83-114 (math.KT/0701778).

[38] P. Seidel, Homological mirror symmetry for the genus two curve, arXiv:0812.1171.

[39] A. Strominger, S.-T. Yau, E. Zaslow, Mirror symmetry is T-duality, Nucl. Phys. B 479 (1996), 243-259 (hep-th/9606040).

[40] R.P. Thomas, Moment maps, monodromy and mirror manifolds, Symplectic geometry and mirror symmetry (Seoul, 2000), World Sci. Publishing, 2001, pp. 467-498 (math.DG/0104196).

[41] R.P. Thomas, S.-T. Yau, Special Lagrangians, stable bundles and mean curvature flow, Comm. Anal. Geom. 10 (2002), 1075-1113 (math.DG/0104197).

Department of Mathematics, M.I.T., Cambridge MA 02139, USA

E-mail address: auroux@math.mit.edu 
